\title{
The Stegana undulata species group (Diptera: Drosophilidae) from the Oriental Region, with molecular phylogenetic analysis of the Chinese species
}

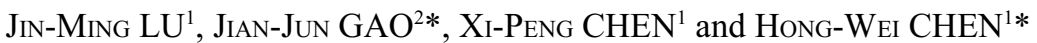 \\ ${ }^{1}$ Department of Entomology, South China Agricultural University, Guangzhou 510642, China \\ ${ }^{2}$ Laboratory for Conservation and Utilization of Bio-resources, Yunnan University, Kunming, Yunnan 650091, China
}

Key words. Drosophilidae, systematics, molecular phylogeny, mitochondrial DNA, Oriental Region, Stegana undulata group, Steganina

\begin{abstract}
The Stegana undulata species group is revised and eight new species described: S. (Steganina) flaviclypeata Chen \& Chen, sp. n., S. (S.) flavipalpata Chen \& Chen, sp. n., S. (S.) leucothorax Chen \& Chen, sp. n., S. (S.) melanocheilota Chen \& Chen, sp. n. and $S$. (S.) melanothorax Chen \& Chen, sp. n. from southern China, and $S$. (S.) flaviscutellata Chen \& Chen, sp. n., $S$. ( $S$.) nigriclypeata Chen \& Chen, sp. n. and $S$. (S.) nigripalpata Chen \& Chen, sp. n. from eastern Malaysia. A key to all the species examined based on morphological data is provided. Based on DNA sequence data of the mitochondrial ND2 and COI genes, the relationship among six Chinese species of the undulata group and that of the $S$. coleoptrata and $S$. nigrolimbata species groups of the same subgenus is investigated, using S. emeiensis of the subgenus Stegana s. str. as an out-group.
\end{abstract}

\section{INTRODUCTION}

The subgenus Steganina Wheeler, 1960 with 113 known species is the biggest subgenus in the genus Stegana Meigen, 1830. Eighty-three species of this subgenus are known from eastern and southeastern Asia (Brake \& Bächli, 2008; Cao \& Chen, 2008; Chen \& Chen, 2008, 2009a, b; Chen et al., 2009; Cheng et al., 2009). The phylogenetic relationships of this subgenus have been analyzed (Okada, 1971; Okada \& Sidorenko, 1992; Sidorenko, 2002; Otranto et al., 2008) and although more than 50 species are still ungrouped, the others have been assigned to five species groups: (1) the coleoptrata group (Laštovka \& Máca, 1982); (2) the nigrolimbata group, or "group 3" in Okada (1971) (Sidorenko, 2002; Cao \& Chen, 2008); (3) the ornatipes group (Cheng et al., 2009); (4) the shirozui group (Chen et al., 2009) and (5) the undulata group, or Okada's (1971) "group 2", with the exclusion of two species, S. nigrifrons de Meijere, 1911 and S. scutellata de Meijere, 1911, and thus, according to Sidorenko (2002), based only on morphological characters of the nominotypical species. This species group is identified based on a combination of the following four characters: (1) face broadened ventrally with conspicuous black band below; (2) 7-13 interfrontal setulae; (3) postpronotal lobe with 2 setae; and (4) hypandrium with 1 pair of paramedian setae (Sidorenko, 2002).

In the present study, eight new species found in South China and East Malaysia are added to the undulata group and the relationships of the undulata group based on DNA sequences of the mitochondrial ND2 and COI genes investigated.

\section{MATERIAL AND METHODS}

Location of type material and morphological terminology

All the type material was collected on tree trunks or tussocks along streams in forests. The specimens are deposited in the following institutions: Institute for Tropical Biology and Conservation, University Malaysia Sabah, Kota Kinabalu, Sabah, Malaysia (ITBC); Kunming Institute of Zoology, Chinese Academy of Sciences, Kunming, China (KIZ); Museum of Kinabalu Park, Sabah Parks, Sabah, Malaysia (KPSP); Museum Zoologicum Bogoriense, Bogor, Indonesia (MZB); Department of Entomology, South China Agricultural University, Guangzhou, China (SCAU); Department of Systematic Entomology, Hokkaido University Museum, Sapporo, Japan (SEHU). The morphological terminology follows McAlpine (1981) and the definitions of measurements, indices and abbreviations, Zhang \& Toda (1992) and Chen \& Toda (2001). H.W. Chen and X.P. Chen described the new species.

\section{Taxa used in the phylogenetic analysis}

Samples of some species of the undulata group, except for those from Malaysia (which had been preserved in Kahle's solution for too long to obtain DNA), were used for DNA sequencing and phylogenetic analysis (Table 1). To test the monophyly of this group, three species, S. nigrolimbata Duda, 1924 (nigrolimbata species group), S. pililobosa Chen \& Chen, 2008 and S. psilolobosa Chen \& Chen, 2008 (both belonging to coleoptrata species group) of the same subgenus Steganina, were included as ingroup taxa. Stegana (Stegana) emeiensis Sidorenko, 1997 was used as the outgroup in the phylogenetic analysis.

\section{DNA extraction, PCR and sequencing}

Total DNA was extracted from a single male using the TIANGENTM DNA extraction kit following the recommended protocol. The ND2 and COI fragments were amplified using the cycle protocol of Zhao et al. (2009). The PCR/sequencing primer pair for the ND2 fragment was 5'-AAGCTACTGGGTTCATACC-3'

\footnotetext{
* Corresponding authors. JJG: gaojj@ynu.edu.cn; HWC: hongweic@scau.edu.cn.
} 
TABLE 1. Details of the samples used in the DNA sequencing and accession numbers of the ND2 and COI sequences; sequences marked with asterisks are those of Li et al. (2010).

\begin{tabular}{|c|c|c|c|c|c|}
\hline \multirow{2}{*}{ Subgenera } & \multirow{2}{*}{ Species group } & \multirow{2}{*}{ Species } & \multirow{2}{*}{ Geographical origin } & \multicolumn{2}{|c|}{ Accession numbers } \\
\hline & & & & ND2 & COI \\
\hline Stegana & & emeiensis & Menglun, Yunnan, China & EU805515* & HM636455 \\
\hline \multirow[t]{12}{*}{ Steganina } & coleoptrata & pililobosa & Shennongjia, Hubei, China & GQ249186 & HM636456 \\
\hline & & psilolobosa & Shennongjia, Hubei, China & GQ249187 & HM636457 \\
\hline & nigrolimbata & nigrolimbata & Guangzhou, Guangdong, China & EU805513* & HM636458 \\
\hline & undulata & melanocheilota & Menglun, Yunnan, China & GQ249188 & HM636459 \\
\hline & & flavipalpata & Nanjian, Yunnan, China & GQ249189 & HM636460 \\
\hline & & flavipalpata & Kunming, Yunnan, China & GQ249190 & HM636461 \\
\hline & & leucothorax & Menglun, Yunnan, China & GQ249191 & HM636462 \\
\hline & & flaviclypeata & Moxi, Sichuan, China & GQ249192 & HM636463 \\
\hline & & flaviclypeata & Jingdong, Yunnan, China & GQ249193 & HM636464 \\
\hline & & melanothorax & Menglun, Yunnan, China & GQ249194 & HM636465 \\
\hline & & undulata & Jianfengling, Hainan, China & GQ249195 & - \\
\hline & & undulata & Mengla, Yunnan, China & GQ249196 & HM636466 \\
\hline
\end{tabular}

(Park, 1999) and 5'-AGGCGATAGATTGTAAATC-3' (He et al., 2009); the PCR/sequencing primer pair for the COI fragment was 5'-CGCCTAAACTTCAGCCACTT-3' (Wang et al., 2006) and 5'-CCTAAATTAGCTCATGTAGAC-3' (He et al., 2009) for $S$. emeiensis and $S$. nigrolimbata, and 5'-GGTCAACAA ATCATAAAGATATTGG-3' and 5'-TAAACTTCAGGGTGA CCAAAAAATCA-3' (Folmer et al., 1994) for the other species. The PCR products were purified and then directly sequenced on an $\mathrm{ABI} 3730$ sequencer.

\section{Sequence aligning and characterization}

The GenBank accession numbers of the sequences used in the present analysis are shown in Table 1. The ND2 sequences of two species, $S$. emeiensis and $S$. nigrolimbata, were obtained from GenBank. The ND2 and COI sequences (Table 1) were aligned using the ClustalW method (Thompson et al., 1994) and the alignments edited manually to make it conform to the codon assignments. The alignments of the two genes were then combined for phylogenetic analyses, with the unavailable $C O I$ sequences for $S$. undulata-HN treated as missing data. Before the phylogenetic analysis, the sequence data were subjected to the base compositional homogeneity test using STATIO (Rzhetsky \& Nei, 1995). The program DAMBE (Xia \& Xie, 2001) was also used to test whether the observed index of substitution saturation (ISS) is significantly lower than the critical index of substitution saturation (ISS.c) (Xia et al., 2003). Models of nucleotide substitution were selected using Modeltest 3.7 (Posada \& Crandall, 1998) for the whole concatenated sequences and partitioned data sets: two data sets partitioned by gene locus (ND2 and COI) and six partitioned by gene locus plus codon position (1st, 2nd and 3rd).

\section{Phylogenetic analysis}

Phylogenetic trees were constructed using the Minimum Evolution (ME) method, program MEGA version 4 (Tamura et al., 2007) and concatenated sequences of the ND2 and COI genes. Based on the result of model selection and the test of base compositional homogeneity using STATIO (Table 2), the Maximum Composite Likelihood model was adopted, with substitution rates across sites set as gamma distributed (shape parameter $=$ $1.1771)$ and nucleotide frequencies among species set as heterogeneous. Bayesian analyses using MrBayes version 3.12 (Ronquist \& Huelsenbeck, 2003) and concatenated sequences were also conducted. For the Bayesian analysis, specific nucleotide substitution models were assigned to data sets partitioned by gene locus only ( 2 data partitions) or by locus and codon position (6 data partitions) according to the results of model selec- tion in Modeltest (Table 2). Two MCMC runs of 1,000,000 generations were conducted in each Bayesian analysis, with a sampling frequency of every 100 generations, and a burning-in of 2,500 early-phase samples for each run. Then a majority-rule tree showing all the compatible partitions was obtained by summarizing the remaining 15,002 samples.

\section{SYSTEMATIC ACCOUNT}

\section{Subgenus Steganina Wheeler, 1960}

Stegana (Steganina) Wheeler, 1960: 110.

Type species: Musca coleoptrata Scopoli, 1763.

Diagnosis. Largest diameter of eye oblique to the body axis; face with black band.

\section{Stegana undulata species group}

Diagnosis (modified from Sidorenko, 2002)

Surstylus not strongly arcuate, mostly with 1 small acute prensiseta submedially on inner margin (absent in $S$. flaviscutellata Chen \& Chen, sp. n.), only distally with dense setae (Figs 4, 9, 13, 18, 22, 27, 32, 41); 10th sternite expanded to lobate distally (Figs 5, 10, 14, 19, 23, 28, 33, 42).

\section{Description (male and female)}

Head: Eyes red. Ocellar triangle black, with a pair of small setae above ocellar setae. Post-vertical setae slightly behind vertex ridge. Frons and face rectangular in lateral view. Frons shiny, with a black band and sporadic, minute setulae below (above ptilinal fissure). Proclinate orbital setae nearer to ptilinal fissure than to inner vertical setae. Face broadened ventrally; facial carina absent. Palpus with 4-5 long setae distally and several shorter setae basally. Gena broad (ch/o $\geq 0.15$ ), whitish yellow. Vibrissa prominent; other orals small. Occiput glossy, yellow, black around occipital foramen.

Thorax: Scutum usually with a few patches and stripes. Mesopleuron with a black longitudinal stripe above (running from propleuron to base of haltere). Postpronotal lobe with 1-3 long and a few small setae. Acrostichal setulae in approximately 10 irregular rows. Prescutellar setae 1 pair. Basal scutellar setae divergent; apical scutellars cruciate. 
TABLE 2. Results of model selection, composition homogeneity test and test of substitution saturation.

\begin{tabular}{|c|c|c|c|c|c|c|c|c|c|c|c|c|}
\hline & \multicolumn{4}{|c|}{ ND2 } & \multicolumn{4}{|c|}{$\mathrm{COI}$} & \multicolumn{4}{|c|}{$\mathrm{ND} 2+\mathrm{COI}$} \\
\hline & $1 \mathrm{st}-\mathrm{CP}^{\mathrm{a}}$ & 2nd-CP & 3rd-CP & All & $1 \mathrm{st}-\mathrm{CP}$ & 2nd-CP & 3rd-CP & All & 1 st-CP & 2nd-CP & 3rd-CP & All \\
\hline \multicolumn{13}{|l|}{ Model selection } \\
\hline Model selected ${ }^{b}$ & $\mathrm{TIM}+\mathrm{G}$ & $\mathrm{HKY}+\mathrm{G}$ & $\mathrm{HKY}+\mathrm{G}$ & $\mathrm{TVM}+\mathrm{G}$ & TrNef $+\mathrm{G}$ & F81 & $\mathrm{HKY}+\mathrm{GC}$ & $\mathrm{GTR}+\mathrm{I}+\mathrm{G}$ & $\mathrm{GTR}+\mathrm{I}+\mathrm{G}$ & $\mathrm{K} 81 \mathrm{uf}+\mathrm{G}$ & $\mathrm{HKY}+\mathrm{G}$ & $\mathrm{GTR}+\mathrm{I}+\mathrm{G}$ \\
\hline freq $\mathrm{A}$ & 0.4023 & 0.2142 & 0.4867 & 0.3574 & - & 0.1545 & 0.4601 & 0.2861 & 0.3389 & 0.1891 & 0.4764 & 0.3338 \\
\hline freq $\mathrm{C}$ & 0.0761 & 0.1770 & 0.0377 & 0.1001 & - & 0.2509 & 0.0525 & 0.1202 & 0.1137 & 0.2081 & 0.0438 & 0.1042 \\
\hline freq $\mathrm{G}$ & 0.0977 & 0.0953 & 0.0168 & 0.0619 & - & 0.1755 & 0.0144 & 0.1686 & 0.1804 & 0.1278 & 0.0159 & 0.1106 \\
\hline freq $\mathrm{T}$ & 0.4239 & 0.5135 & 0.4579 & 0.4806 & - & 0.4191 & 0.4730 & 0.4251 & 0.3770 & 0.4750 & 0.4640 & 0.4513 \\
\hline $\mathrm{Ti} / \mathrm{tv}$ ratio & - & 1.2708 & 6.5309 & - & - & - & 8.3515 & - & - & - & 6.2401 & - \\
\hline $\mathrm{R}[\mathrm{A}-\mathrm{C}]$ & 1.0000 & - & - & 2.3944 & 1.0000 & - & - & 0.0000 & 1.7093 & 1.0000 & - & 5.9087 \\
\hline$R[A-G]$ & 7.8962 & - & - & 27.3988 & 1.1580 & - & - & 23.8169 & 7.4587 & 4.3910 & - & 41.6883 \\
\hline $\mathrm{R}[\mathrm{A}-\mathrm{T}]$ & 4.2531 & - & - & 8.2086 & 1.0000 & - & - & 57.1175 & 7.9487 & 2.6794 & - & 39.1278 \\
\hline $\mathrm{R}[\mathrm{C}-\mathrm{G}]$ & 4.2531 & - & - & 5.9980 & 1.0000 & - & - & 0.0000 & 0.0000 & 2.6794 & - & 10.3767 \\
\hline $\mathrm{R}[\mathrm{C}-\mathrm{T}]$ & 27.9204 & - & - & 27.3988 & 82.6756 & - & - & 277.8802 & 65.9865 & 4.3910 & - & 174.6169 \\
\hline $\mathrm{R}[\mathrm{G}-\mathrm{T}]$ & 1.0000 & - & - & 1.0000 & 1.0000 & - & - & 1.0000 & 1.0000 & 1.0000 & - & 1.0000 \\
\hline Proportion of invariable sites (I) & 0 & 0 & 0 & 0 & 0 & 0 & 0 & 0.6694 & 0.6316 & 0 & 0 & 0.5407 \\
\hline Gamma distr. shape parameter & 0.2378 & 0.2163 & 0.4522 & 0.2867 & 0.1344 & - & 0.3568 & 2.6757 & 0.1665 & 0.0142 & 0.4236 & 1.1771 \\
\hline \multicolumn{13}{|l|}{ Composition homogeneity test } \\
\hline$d f$ & 36 & 36 & 36 & 36 & 33 & 33 & 33 & 33 & 33 & 33 & 33 & 33 \\
\hline$I$ & 30.73 & 18.83 & 64.55 & 49.44 & 34.55 & 25.33 & 2.03 & 34.47 & 45.98 & 39.96 & 17.78 & 49.67 \\
\hline$P(\%)$ & 71.73 & 99.18 & 0.24 & 6.72 & 39.37 & 82.76 & 100.00 & 39.74 & 6.61 & 18.84 & 98.58 & 3.14 \\
\hline \multicolumn{13}{|l|}{ Test of substitution saturation } \\
\hline Iss & 0.3043 & 0.0650 & 0.3865 & 0.1738 & 0.0668 & 0.0191 & 0.4492 & 0.3878 & 0.3989 & 0.1448 & 0.5325 & 0.4530 \\
\hline Iss.c (for an asymmetrical tree) & 0.7010 & 0.7047 & 0.7010 & 0.7631 & 0.6975 & 0.7791 & 0.6974 & 0.7447 & 0.7264 & 0.7293 & 0.7263 & 0.7952 \\
\hline$P$ & 0.0000 & 0.0000 & 0.0000 & 0.0000 & 0.0000 & 0.0000 & 0.0000 & 0.0000 & 0.0000 & 0.0000 & 0.0000 & 0.0000 \\
\hline Iss.c (extremely asymm. tree) & 0.5193 & 0.5346 & 0.5193 & 0.5974 & 0.5378 & 0.7450 & 0.5376 & 0.5949 & 0.5508 & 0.5649 & 0.5507 & 0.6411 \\
\hline$P$ & 0.0000 & 0.0000 & 0.0000 & 0.0000 & 0.0000 & 0.0000 & 0.0038 & 0.0000 & 0.0000 & 0.0000 & 0.3736 & 0.0000 \\
\hline
\end{tabular}

Wing (Fig. 1): Dark brown anteriorly, paler posteriorly and curved downward over most of its length. Basal medial-cubital cross vein present; $\mathrm{C}_{1}$ with 2 subequal setae. Costal vein with 6-8 minute spinules on ventral surface between veins $R_{2+3}$ and $R_{4+5}$. Vein $R_{2+3}$ markedly curved to costa at tip; veins $R_{4+5}$ and $M_{1}$ convergent distally (Fig. 1). Halteres white basally, grey-brown distally.

Legs: Fore femur with 1 row of setae on posterior surface and 1 row of 3-8 setae on ventral surface. Mid tibia with 4-6 strong, suberect setae on basal part of dorsal surface. Apical seta present on mid tibia. Preapical dorsal setae present on all tibiae. Mid and hind tarsomeres with 2 and 1 row(s) of minute cuneiform setulae on ventral surface, respectively; fore and hind first tarsomeres slightly shorter than the rest combined; mid first tarsomere longer than the rest combined.

Abdominal sternites broad, wider than long.

Male terminalia: Epandrium pubescent, mostly with dense setae near posterior margin on each side (Figs 3, 8, $13,17,22,26,31,36,40)$. Surstylus separated from epandrium. Cercus separated from epandrium, setigerous, but not pubescent (Figs 3, 8, 13, 17, 22, 26, 31, 36, 40). Tenth sternite connected with surstyli basolaterally. Hypandrium anteromedially elongated (Figs 6, 7, 11, 12, $15,16,20,21,24,25,29,30,34,35,38,39,43,44$ ), with the pair of large membranous flaps fused to each other, sometimes with a few paramedian setae posteroventrally (Figs 15, 16, 24, 25). Parameres (paraphyses) absent (Figs $6,7,11,12,15,16,20,21,24,25,29,30,34,35,38,39$, 43, 44). Gonopods (dorsal arch in Bächli et al., 2004) coalesce, forming posteromedian lobe, slightly vaulted, basolaterally contiguous to posterior ends of hypandrium (Figs 5, 10, 14, 19, 23, 28, 33, 37, 42). Aedeagus broad distally, with tentacle-like setae (marginal fringe in Bächli et al., 2004) on apical margin, basally fused with slender aedeagal apodeme (Figs 6, 7, 11, 12, 15, 16, 20, 21, 24, $25,29,30,34,35,38,39,43,44)$.

\section{Stegana (Steganina) undulata de Meijere, 1911}

(Figs 1-7)

Stegana undulata de Meijere, 1911: 419. Type locality: Indonesia (Java).

Stegana (Steganina) undulata de Meijere: Okada, 1971: 87; Okada \& Sidorenko, 1992: 424; Sidorenko, 1998: 296.

Stegana (Stegana) undulata de Meijere: Sidorenko \& Okada, 1991: 659.

Diagnosis. Veins $\mathrm{R}_{4+5}$ and $\mathrm{M}_{1}$ with several setae on basal section of dorsal surface (Figs 1,2); distal part of 10th sternite nearly rhomboid, about 1.9 times as wide as long (Fig. 5).

Material examined. CHINA: $1 \delta^{\hat{}}$ (SCAU, No. 120112), Fusui, Chongzuo, Guangxi, $22^{\circ} 31^{\prime} \mathrm{N}, 107^{\circ} 40^{\prime} \mathrm{E}, 230 \mathrm{~m}$, 18.viii.2004, tree trunks, H.W. Chen; $7 \hat{\jmath}, 89$ (SCAU, Nos 120113-22), Diaoluoshan, Lingshui, Hainan, $18^{\circ} 10^{\prime} \mathrm{N}$, $108^{\circ} 52^{\prime} \mathrm{E}, 1,000 \mathrm{~m}$, 3.xii.2003, tree trunks, H.W. Chen; $3{ }^{\star}, 1$ ㅇ (SCAU, Nos 120123-25), Jianfengling, Ledong, Hainan, $18^{\circ} 41^{\prime} \mathrm{N}, 108^{\circ} 52^{\prime} \mathrm{E}, 900 \mathrm{~m}, 30 . x i .2003$, 19.v.2004, tree trunks, H.W. Chen; 420ิ, 6 ㅇ (SCAU, Nos 120126-70), Mengla, Xishuangbanna, Yunnan, $21^{\circ} 28^{\prime} \mathrm{N}, 101^{\circ} 38^{\prime} \mathrm{E}, 680 \mathrm{~m}$, 10.-12.ix.2002, 21.-24.iv.2007, tree trunks and tussock, H.W. Chen, J.J. Gao, F. Zhao; $140^{\Uparrow}, 10$ 우 (KIZ), Menglun, Xishuangbanna, Yunnan, $21^{\circ} 41^{\prime} \mathrm{N}, 101^{\circ} 25^{\prime} \mathrm{E}, 700 \mathrm{~m}, 24 .-26 . x i i .2003$, 16.-20.iv.2007, tree trunks, H.W. Chen, J.J. Gao, F. Zhao; $2 \delta^{\hat{\sigma}}$, 47 우 (SCAU, Nos 120171-200), Mengyang, Xishuangbanna, 

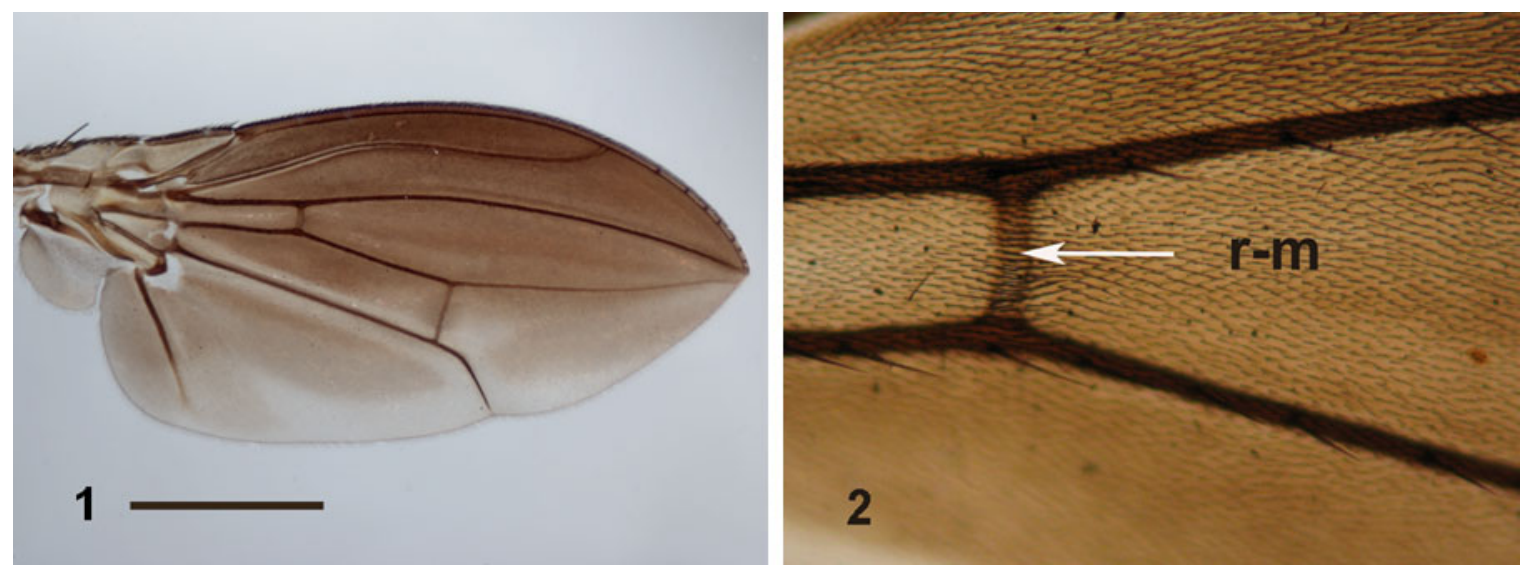

Figs 1, 2. Stegana (Steganina) undulata de Meijere, $₫$, right wing dorsal. 1 - overview, scale bar = $1 \mathrm{~mm} ; 2$ - basal section of $\mathrm{R}_{4+5}$ and $\mathrm{M}_{1}$, each bearing several setae.

Yunnan, $22^{\circ} 20^{\prime} \mathrm{N}, 100^{\circ} 51^{\prime} \mathrm{E}, 700 \mathrm{~m}, 14 .-15 . x i .2002$, tree trunks, H.W. Chen. MYANMAR: $10^{\star}$ (SEHU), Rangoon (Yan-

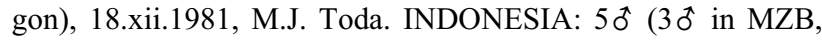
20 in SEHU), Bogor, Java, 8.xii.1996, underside of fallen logs, M.J. Toda.

Distribution. China (Guangxi, Hainan, Yunnan), Myanmar (Yangon), Indonesia (Java, Sumatra, Kalimantan).

\section{Stegana (Steganina) melanocheilota Chen \& Chen,} sp. n.

(Figs 8-12)

Diagnosis. Different from S. undulata in that the distal part of 10th sternite is strongly broadened, transversely oval, about 1.9 times as wide as long (Fig. 10) and the distal part of aedeagus slightly rounded in ventral view (Fig. 11).

Description. Male and Female: Frons orange yellow, medially brown. Fronto-orbital plate orange yellow. Pedicel yellow; first flagellomere almost black. Face black on upper $1 / 2$, yellow on lower $1 / 2$. Clypeus black. Palpus yellow basally, black distally. Mesonotum yellowish brown, with 2 pairs of yellow longitudinal stripes submedially and a few grey-yellow patches laterally (anterior to wing base). Postpronotal lobe brown on upper $1 / 5$, white on lower $4 / 5$. Katepisternum white. Scutellum brown. Costal vein with 8-10 minute spinules on ventral surface between veins $\mathrm{R}_{2+3}$ and $\mathrm{R}_{4+5}$. Legs brown, white on basal parts of all femora and distal part of mid tibia,
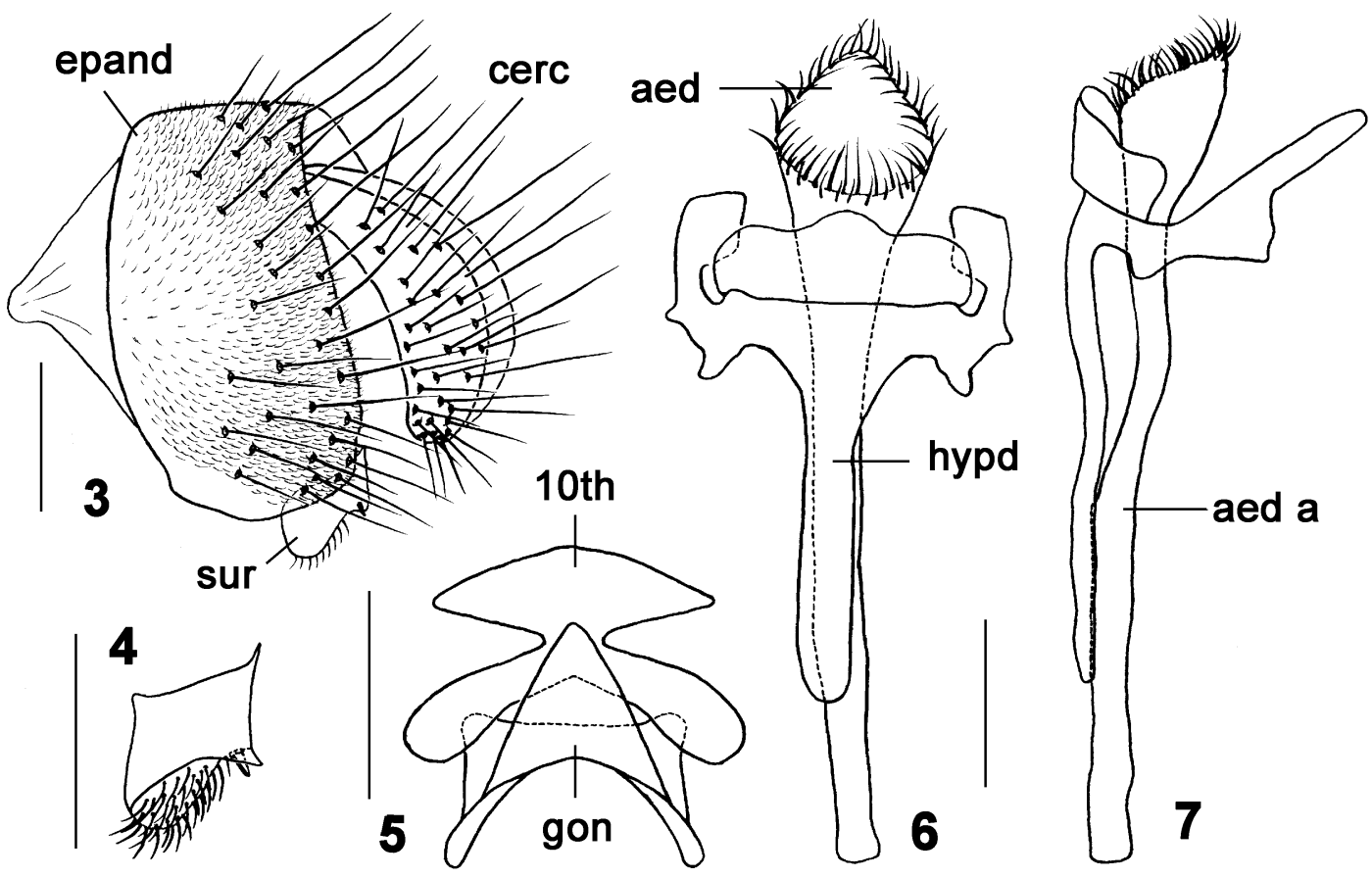

Figs 3-7. Stegana (Steganina) undulata de Meijere, đิ. 3 - epandrium (epand), surstylus (sur) and cercus (cerc) (lateral view); 4 surstylus; 5 - 10th sternite (10st) and gonopods (gon) (ventral view); 6 - hypandrium (hypd), aedeagus (aed) and aedeagal apodeme (aed a) (ventral view); 7 - ditto (lateral view). Scale bars $=0.1 \mathrm{~mm}$. 


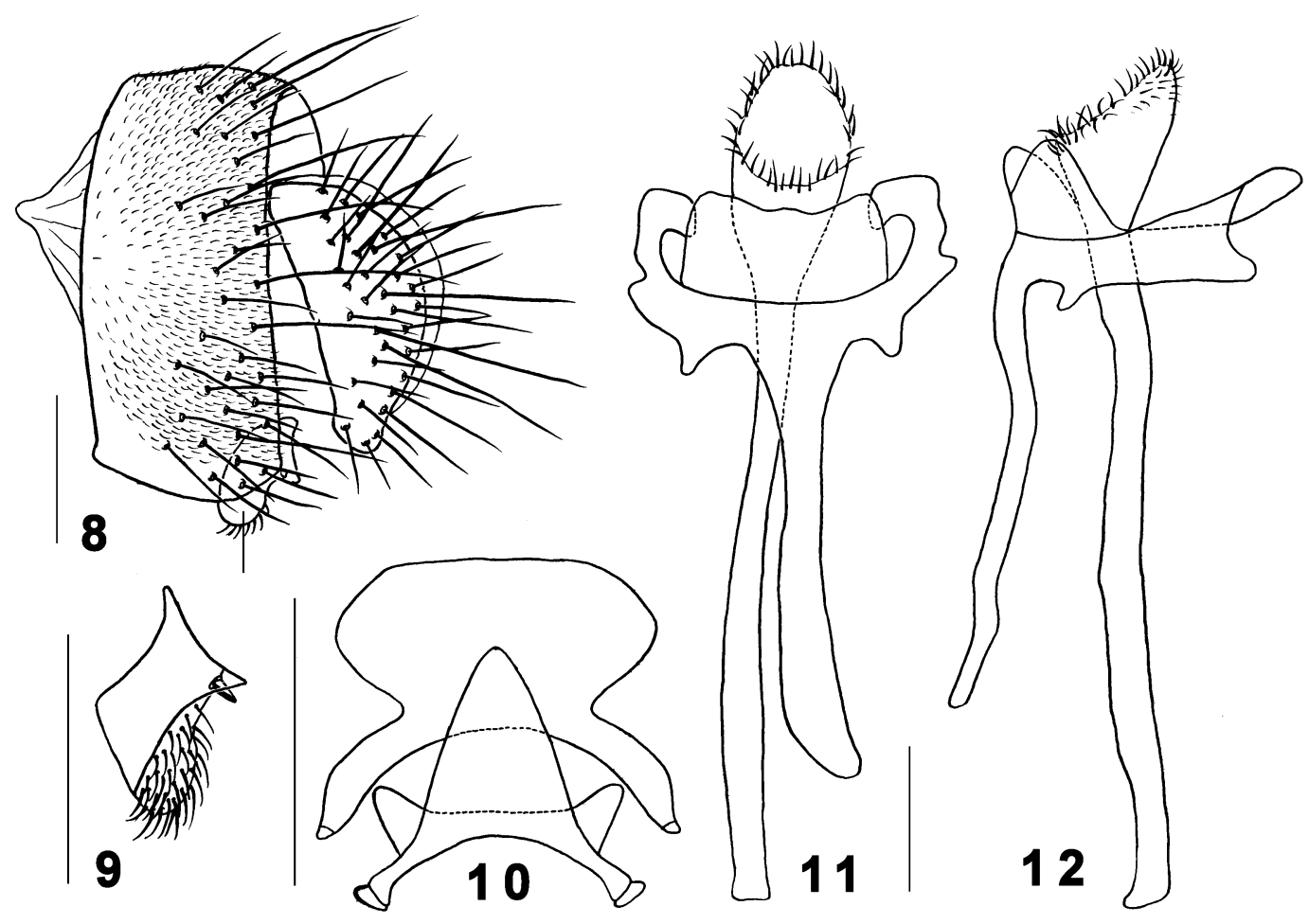

Figs 8-12. Stegana (Steganina) melanocheilota Chen \& Chen, sp. n., ô. 8 - epandrium, surstylus and cercus; 9 - surstylus; 10 10 th sternite and gonopods; 11 - hypandrium, aedeagus and aedeagal apodeme; 12 - ditto. For orientation, see Figs 3-7. Scale bars $=0.1 \mathrm{~mm}$.

black on fore and mid knees. Fore femur with 6 setae on distal part of ventral surface. Abdominal 1 st to 5 th tergites black medially, yellow laterally; 6th tergite nearly black. Sternites brown.

Male terminalia: Hypandrium anteromedially remarkably elongated, 2.4 times longer than width of epandrium and lacking paramedian setae (Figs 11, 12). Gonopods slightly triangular (Fig. 10). Aedeagus about 0.25 times as long as aedeagal apodeme (Fig. 12).

Measurements. $\mathrm{BL}=3.45 \mathrm{~mm}$ in holotype (range in $2 \widehat{ } \widehat{ } \mathrm{t}$ and 3 paratypes: $3.35-3.85 \mathrm{~mm}$ in $\widehat{\sigma}, 3.20-3.70 \mathrm{~mm}$ in 9 ), $\mathrm{ThL}=$ $1.68 \mathrm{~mm}(1.80-1.90 \mathrm{~mm}$ in 0 , $1.68-1.19 \mathrm{~mm}$ in ㅇ $), \mathrm{WL}=3.05$

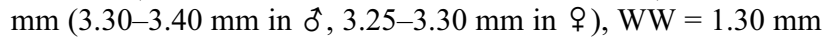
$(1.15-1.30 \mathrm{~mm}$ in $\delta, 1.05-1.37 \mathrm{~mm}$ in $\$)$, arb $=11 / 8$ $(10-11 / 7-8)$, avd $=0.77(0.71-0.85)$, adf $=1.63(1.50-2.14)$, flw $=1.75(1.75-2.14), \mathrm{FW} / \mathrm{HW}=0.41(0.40-0.44), \mathrm{ch} / \mathrm{o}=0.15$ $(0.15-0.18)$, prorb $=0.88(0.89-0.94)$, rcorb $=0.75(0.77-0.89)$, $\mathrm{vb}=0.63(0.59-0.73), \mathrm{dcl}=0.47(0.48), \mathrm{sctl}=1.37$, sterno $=$ $0.95(0.81-0.94)$, orbito $=2.20(1.83-2.20), \mathrm{dcp}=0.21$ (0.19-0.24), sctlp $=2.20(1.91-2.30), \mathrm{C}=1.92(1.88-2.20), 4 \mathrm{c}$ $=1.15(0.97-2.23), 4 \mathrm{v}=1.79(1.63-2.00), 5 \mathrm{x}=1.50$ (1.33-1.62), ac $=9.50(8.75-10.25), \mathrm{M}=0.55(0.44-0.68), \mathrm{C} 3 \mathrm{~F}$ $=0.70(0.65-0.74)$.

Type material. Holotype ơ (SCAU, No. 120201), CHINA: Menglun, Xishuangbanna, Yunnan, 17.iv.2007, ex tussock, J.J. Gao. Paratypes: CHINA: $2 \hat{\sigma}, 3 q(10,1 \%$ in KIZ, $10,2 q$ in SCAU, Nos 120202-04), same data as holotype, 17.-18.iv.2007, tree trunks and tussock, H.W. Chen, J.J. Gao, F. Zhao.

Etymology. A combination of the Greek words melas + cheilos referring to the black clypeus.

Distribution. China (Yunnan).

\section{Stegana (Steganina) flavipalpata Chen \& Chen, sp. n.}

(Figs 13-16)

Diagnosis. Differs from other species of the undulata group in the yellow scutellum with brown stripes or patches in the male, dark brown with yellow patch at tip in female, and numerous serrated, minute processes at the base of the aedeagus (Fig. 16).

Description. Male and female. Frons black, with orange yellow band submedially. Fronto-orbital plate orange yellow. Pedicel black basally, orange yellow distally; first flagellomere almost black. Face black on upper $1 / 2$, yellow on lower 1/2. Clypeus yellow. Palpus yellow. Mesonotum yellowish brown, with a broad black stripe medially and a few brown patches laterally (anterior to wing base). Postpronotal lobe white on anterior $1 / 2$, brown on posterior $1 / 2$. Katepisternum white. Costal vein with 8-11 minute spinules on ventral surface between veins $R_{2+3}$ and $R_{4+5}$. Veins $R_{4+5}$ and $M_{1}$ convergent distally, as in S. undulata (Fig. 1). Legs white with black knee articulations. Fore femur with 8 setae on ventral surface. Mid tibia with 5 strong setae on basal part of dorsal surface. Abdominal tergites black except for 2nd and 3rd tergites yellow laterally. Sternites dark brown.

Male terminalia: Hypandrium anteromedially elongated, about 1.28 times as long as width of epandrium and with a few paramedian setae posteroventrally (Figs 15, 16). Distal part of 10th sternite umbrella-like, about 2.4 times as wide as long (Fig. 14). Gonopods slightly rounded posteromedially (Fig. 14). Aedeagus about 0.63 times as long as aedeagal apodeme (Fig. 16). 


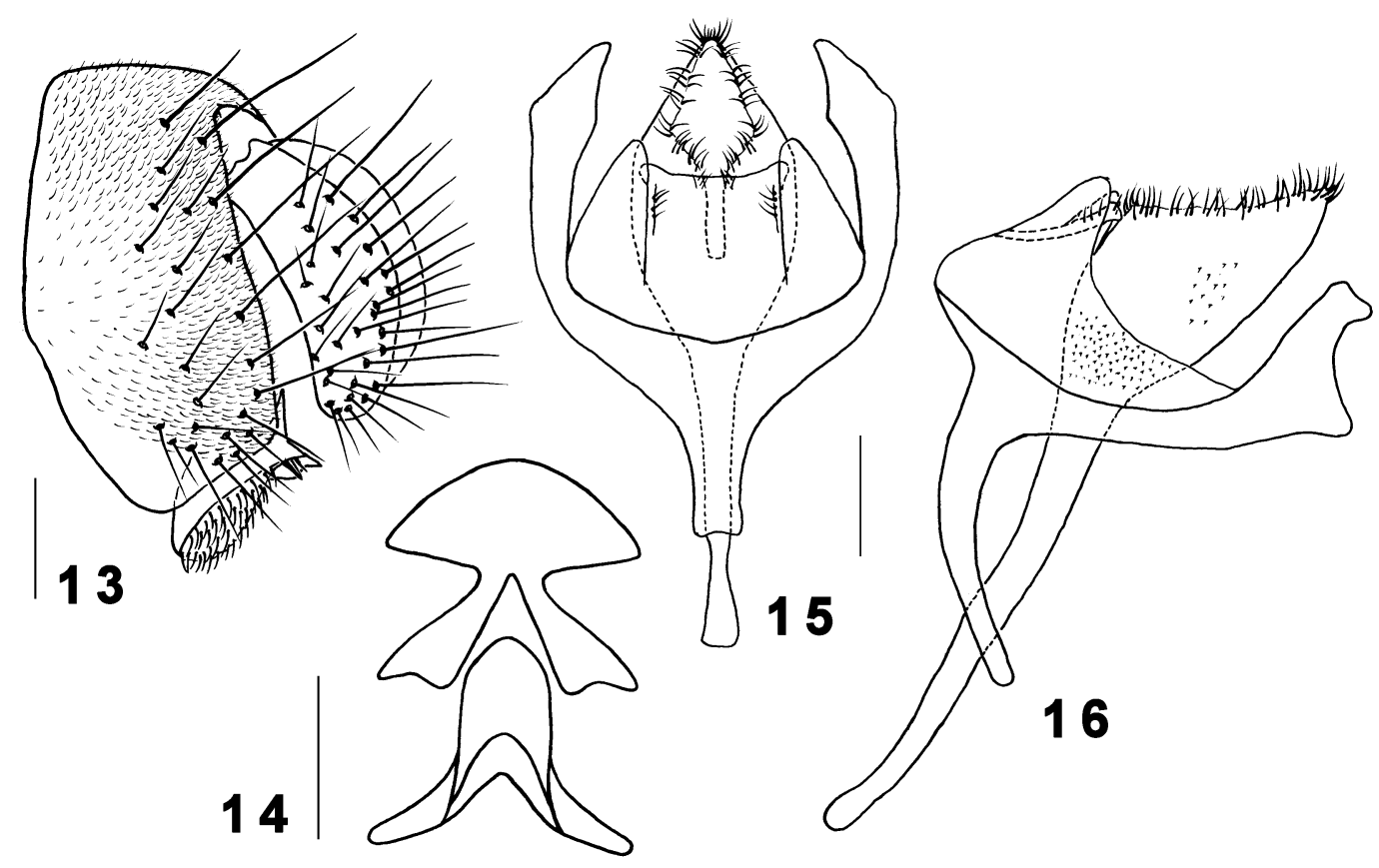

Figs 13-16. Stegana (Steganina) flavipalpata Chen \& Chen, sp. n., ô. 13 - epandrium, surstylus and cercus; 14 - 10th sternite and gonopods; 15 - hypandrium, aedeagus and aedeagal apodeme; 16 - ditto. For orientation, see Figs 3-7. Scale bars $=0.1 \mathrm{~mm}$.

Measurements. $\mathrm{BL}=3.67 \mathrm{~mm}$ in holotype (range in $4 \hat{0}$ and 3 p paratypes: $3.40-3.75 \mathrm{~mm}$ in $\widehat{0}, 3.00-4.00 \mathrm{~mm}$ in ㅇ), $\mathrm{ThL}=$ $2.22 \mathrm{~mm}(2.20-2.35 \mathrm{~mm}$ in $0,2.03-2.24 \mathrm{~mm}$ in ㅇ $), \mathrm{WL}=4.20$ $\mathrm{mm}(4.20-4.55 \mathrm{~mm}$ in $\delta, 3.75-4.30 \mathrm{~mm}$ in ㅇ $), \mathrm{WW}=1.55 \mathrm{~mm}$ $\left(1.63-1.90 \mathrm{~mm}\right.$ in $\delta^{\star}, 1.53-1.70 \mathrm{~mm}$ in $\left.९\right)$, arb $=9 / 7(9 / 6-7)$, avd $=0.79(0.71-0.85)$, adf $=1.75(1.44-2.00)$, flw $=2.00$ $(1.50-2.12), \quad \mathrm{FW} / \mathrm{HW}=0.42(0.42-0.51), \quad \mathrm{ch} / \mathrm{o}=0.18$ $(0.15-0.20)$, prorb $=1.12(0.85-1.12)$, rcorb $=0.82(0.73-0.86)$, $\mathrm{vb}=0.62(0.54-0.74), \mathrm{dcl}=0.58(0.41-0.56)$, sctl $=1.22$ $(1.25-1.57)$, sterno $=0.77(0.64-0.86)$, orbito $=1.83$ $(1.83-2.20), \mathrm{dcp}=0.26(0.26-0.29), \mathrm{sctlp}=1.67(1.50-2.00), \mathrm{C}$ $=1.61(1.58-1.76), 4 \mathrm{c}=1.50(1.16-1.50), 4 \mathrm{v}=1.82$ (1.70-1.95), $5 \mathrm{x}=1.46(1.22-1.47), \mathrm{ac}=10.20(9.80-10.60), \mathrm{M}$ $=0.56(0.42-0.61), \mathrm{C} 3 \mathrm{~F}=0.74(0.71-0.78)$.

Type material. Holotype ${ }^{\star}$ (SCAU, No. 120205), CHINA: Wuliangshan, Nanjian, Yunnan, $24^{\circ} 31^{\prime} \mathrm{N}, 101^{\circ} 40^{\prime} \mathrm{E}, 2,230 \mathrm{~m}$, 25.vii.2006, tree trunk, T. Li. Paratypes: CHINA: 10 (SCAU, No. 120206), same data as holotype; $20,3+(10,1 \%$ in KIZ, 10,29 in SCAU, Nos 120207-09), Wuliangshan, Jingdong, Yunnan, 4.-5.viii.2006, tree trunks, H.L. Cao, H.Z. Cao, T. Li; 1 đ (SCAU, No. 120210), Jiaoye Park (Qiongzhusi), Kunming, Yunnan, 19.viii.2006, tree trunk, H.L. Cao.

Etymology. A combination of the Latin words flavus + palpus, referring to the yellow palpus.

Distribution. China (Yunnan).

\section{Stegana (Steganina) leucothorax Chen \& Chen, sp. n.} (Figs 17-21)

Diagnosis. Differs from $S$. melanocheilota as it has a yellow clypeus and the distal part of 10th sternite slightly transversely oval, 1.7 times as wide as long (Fig. 19).

Description. Male and female. Frons orange yellow, with black stripes sublaterally. Fronto-orbital plate orange yellow. Pedicel yellow; first flagellomere almost black, greyish yellow basally. Face black on upper $1 / 2$, yellow on lower $1 / 2$. Palpus yellow basally, black distally. Mesonotum brown, with 1 yellow stripe medially and 3 pairs of dark brown stripes submedially and a few greyish yellow patches laterally (anterior to wing base). Postpronotal lobe brown on anterior $2 / 5$, white on posterior $3 / 5$. Katepisternum white. Scutellum brown. Costal vein with 8-10 minute spinules on ventral surface between veins $\mathrm{R}_{2+3}$ and $\mathrm{R}_{4+5}$. Legs brown, white on basal parts of all femora and distal part of mid tibia, black on fore and mid knees. Abdominal tergites 1 to 5 black medially, yellow laterally; 6th tergite nearly black. Sternites dark brown.

Male terminalia: Hypandrium anteromedially elongated, about 2.47 times as long as width of epandrium, without paramedian setae posteroventrally (Figs 20, 21). Gonopods shovel-like and acute apically (Fig. 19). Distal part of aedeagus triangular in ventral view (Fig. 20), without serrated, minute processes, about 0.3 times as long as aedeagal apodeme (Fig. 21).

Measurements. $\mathrm{BL}=3.00 \mathrm{~mm}$ in holotype (range in $4 \sigma^{\circ}$ and 5 paratypes: $2.75-3.15 \mathrm{~mm}$ in $\hat{\delta}, 2.90-3.25 \mathrm{~mm}$ in 9 ), $\mathrm{ThL}=$ $1.70 \mathrm{~mm}(1.56-1.75 \mathrm{~mm}$ in $\delta, 1.64-1.86 \mathrm{~mm}$ in $९), \mathrm{WL}=2.85$ $\mathrm{mm}(2.90-3.05 \mathrm{~mm}$ in $\delta, 2.30-3.20 \mathrm{~mm}$ in + ), $\mathrm{WW}=1.36 \mathrm{~mm}$ $(0.96-1.28 \mathrm{~mm}$ in $\delta, 1.10-1.32 \mathrm{~mm}$ in + ), arb $=13 / 7$ (11-13/7-8), avd $=0.77(0.77-0.85)$, adf $=2.17(1.63-2.00)$, $\mathrm{flw}=2.50(1.85-2.50), \mathrm{FW} / \mathrm{HW}=0.39(0.43-0.47), \mathrm{ch} / \mathrm{o}=0.15$ $(0.15-0.16)$, prorb $=1.00(0.88-1.00)$, rcorb $=0.94(0.81-0.88)$, $\mathrm{vb}=0.63(0.56-0.75), \mathrm{dcl}=0.50(0.39-0.59)$, sctl $=1.60$ $(1.40-1.70), \quad$ sterno $=0.90(0.81-0.91)$, orbito $=2.20$ $(1.83-2.50), \mathrm{dcp}=0.26(0.23-0.30)$, sctlp $=2.00(1.73-2.11), \mathrm{C}$ $=1.87(1.76-2.16), 4 \mathrm{c}=1.18(1.03-1.19), 4 \mathrm{v}=1.70$ (1.65-1.79), $5 \mathrm{x}=1.31(1.07-1.45), \mathrm{ac}=13.00(11.00-13.67), \mathrm{M}$ $=0.52(0.47-0.56), \mathrm{C} 3 \mathrm{~F}=0.78(0.76-0.78)$.

Type material. Holotype ơ (SCAU, No. 120211), CHINA: Wangtianshu, Mengla, Xishuangbanna, Yunnan, 10.ix.2002, tree trunk, H.W. Chen. Paratypes: CHINA: $15 \hat{\delta}, 15 q(5 \delta, 5 q$ in KIZ, $80,8 \%$ in SCAU, Nos 120212-27, $20,2 q$ in SEHU), same data as holotype, 10.-11.ix.2002, 21.-25.iv.2007, tree trunks and tussock, H.W. Chen, J.J. Gao, F. Zhao; 30 (SCAU, 


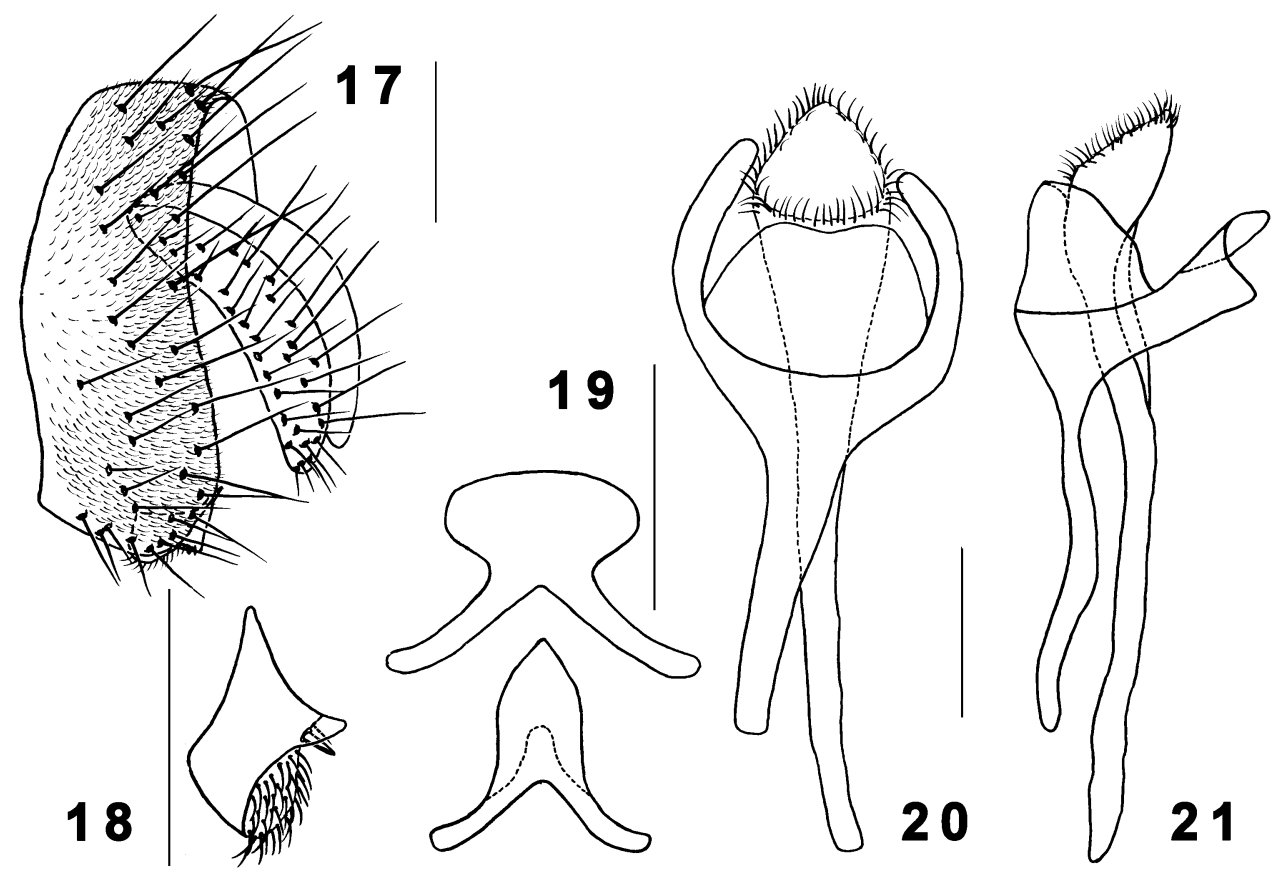

Figs 17-21. Stegana (Steganina) leucothorax Chen \& Chen, sp. n., ô. 17 - epandrium, surstylus and cercus; 18 - surstylus; 19 10 th sternite and gonopods; 20 - hypandrium, aedeagus and aedeagal apodeme; 21 - ditto. For orientation, see Figs 3-7. Scale bars $=0.1 \mathrm{~mm}$.

Nos 120228-30), Menglun, Xishuangbanna, Yunnan, 13.-14.ix.2002, tree trunks, H.W. Chen; 140,12 우 (SCAU, Nos 120231-56), Mengyang, Xishuangbanna, Yunnan, 14.-15.ix.2002, tree trunks, H.W. Chen.

Etymology. A combination of the Greek words leucon + thorax, referring to the white pleura.

Distribution. China (Yunnan).

\section{Stegana (Steganina) flaviclypeata Chen \& Chen, sp. n.}

(Figs 22-25)

Diagnosis. Separated from S. flavipalpata by having a yellow clypeus, the scutellum dark brown, yellow at tip, the distal part of 10th sternite nearly rounded, 0.85 times as wide as long (Fig. 23), and the aedeagus lacking serrated minute processes (Fig. 25).

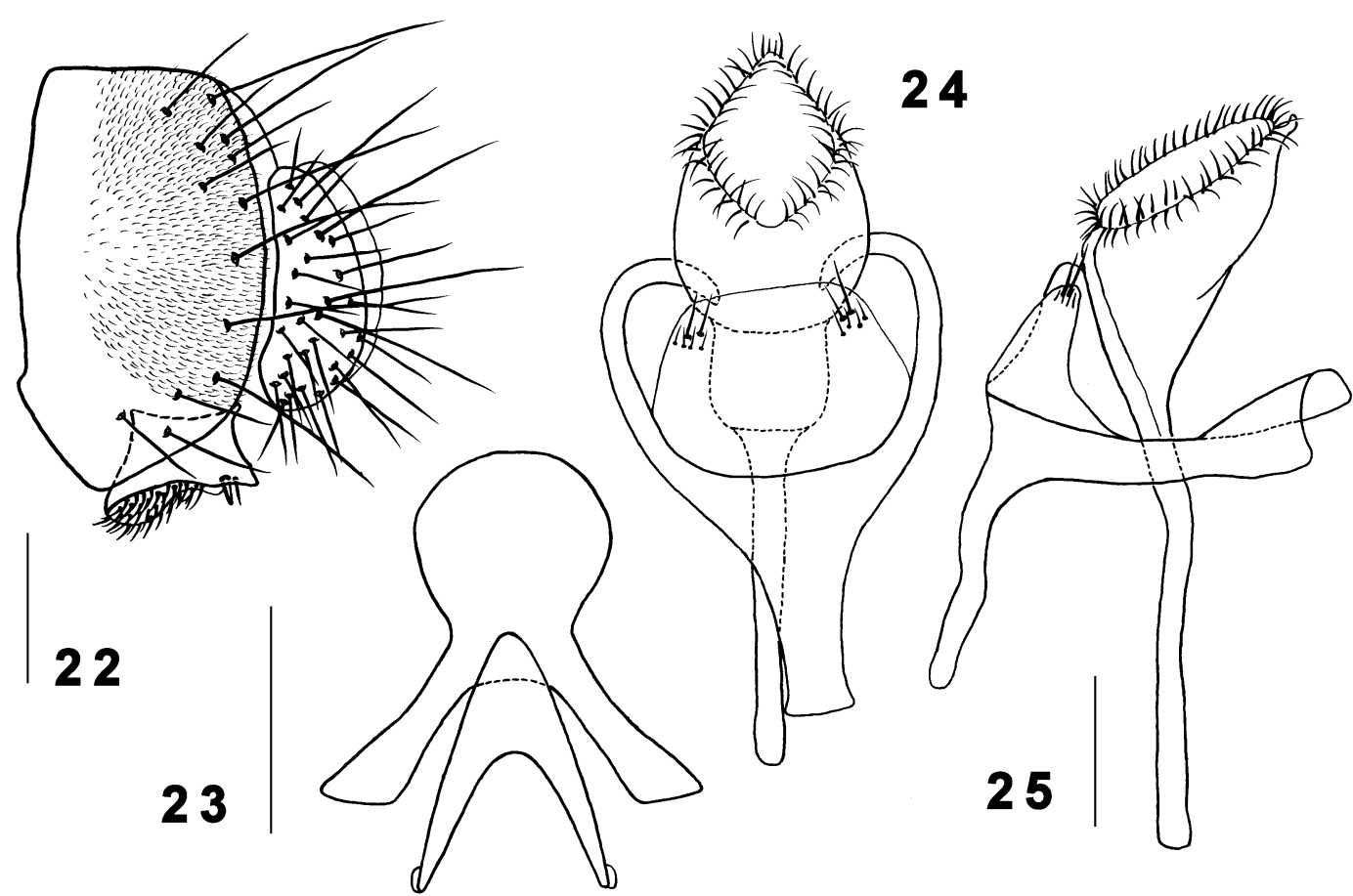

Figs 22-25. Stegana (Steganina) flaviclypeata Chen \& Chen, sp. n., §. 22 - epandrium, surstylus and cercus; 23 - 10th sternite and gonopods; 24 - hypandrium, aedeagus and aedeagal apodeme; 25 - ditto. For orientation, see Figs 3-7. Scale bars $=0.1 \mathrm{~mm}$. 




Figs 26-30. Stegana (Steganina) flaviscutellata Chen \& Chen, sp. n., ô. 26 - epandrium, surstylus and cercus; 27 - surstylus; 28 - 10th sternite and gonopods; 29 - hypandrium, aedeagus and aedeagal apodeme; 30 - ditto. For orientation, see Figs 3-7. Scale bars $=0.1 \mathrm{~mm}$.

Description. Male. Frons orange yellow, with black stripes sublaterally. Fronto-orbital plate orange yellow. Pedicel and first flagellomere yellow, with black pubescence along margin. Face black, with yellow band medially. Palpus yellow. Mesonotum brown to dark brown, with yellow stripes and patches laterally (anterior to wing bases). Postpronotal lobe whitish yellow on anterior $2 / 5$, brown on posterior $3 / 5$. Katepisternum whitish yellow. Costal vein with 9-11 minute spinules on ventral surface between veins $R_{2+3}$ and $R_{4+5}$. Legs mostly white, all knee articulations black. Abdominal tergites 1 to 5 brown medially, pale laterally; 6th tergite nearly black. Sternites yellow, slightly brownish laterally.

Male terminalia: Hypandrium anteromedially slightly elongated, about 1.2 times as long as width of epandrium, with a few paramedian setae posteroventrally (Figs 24, 25 ). Gonopods nearly triangular and slightly rounded apically (Fig. 23). Aedeagus very expanded apically in ventral view, about 0.63 times as long as aedeagal apodeme (Figs 24, 25).

Measurements. $\mathrm{BL}=3.47 \mathrm{~mm}$ in holotype, $\mathrm{ThL}=1.85 \mathrm{~mm}$, $\mathrm{WL}=3.88 \mathrm{~mm}, \mathrm{WW}=1.40 \mathrm{~mm}$, arb $=7 / 6$, avd $=0.90$, adf $=$ 1.25, flw $=1.75, \mathrm{FW} / \mathrm{HW}=0.47, \mathrm{ch} / \mathrm{o}=0.21$, prorb $=1.17$, $\mathrm{rcorb}=0.83, \mathrm{vb}=0.50, \mathrm{dcl}=0.52, \mathrm{sctl}=1.32$, sterno $=0.86$, orbito $=1.67, \mathrm{dcp}=0.31, \mathrm{sctlp}=1.90, \mathrm{C}=2.70,4 \mathrm{c}=1.05,4 \mathrm{v}=$ $1.61,5 \mathrm{x}=1.47, \mathrm{ac}=9.20, \mathrm{M}=0.50, \mathrm{C} 3 \mathrm{~F}=0.73$.

Type material. Holotype ot (SCAU, No. 120257), CHINA: Moxi, Luding, Sichuan, $29^{\circ} 39^{\prime} \mathrm{N}, 102^{\circ} 06^{\prime} \mathrm{E}, 1,100 \mathrm{~m}$, 17.ix.2005, tree trunks, H.W. Chen. Paratypes: 10 , 19 (SCAU, Nos 120258, 59), Wuliangshan, Jingdong, Simao, Yunnan, 4.-5.viii.2006, tree trunks, H.L. Cao, H.Z. Cao.

Etymology. A combination of the Latin words flavus + clypeus, referring to the yellow clypeus.

\section{Distribution. China (Sichuan, Yunnan).}

Stegana (Steganina) flaviscutellata Chen \& Chen, sp. n. (Figs 26-30)

Diagnosis. Differs from other species of the undulata group in having a surstylus without prensiseta (Fig. 27) and in the distal part of 10th sternite infundibular in ventral view (Fig. 28).

Description. Male and female. Frons and fronto-orbital plate orange yellow. Pedicel orange yellow; basal $1 / 2$ of first flagellomere yellow, distal 1/2 black. Face yellow, black on lower margin. Clypeus black. Palpus yellow basally, black distally. Mesonotum yellow, with dark brown stripes and patches laterally. Postpronotal lobe yellow on anterior $3 / 5$, brown on posterior $2 / 5$. Katepisternum brown, slightly yellowish ventrally. Scutellum yellow. Costal vein with 9-11 minute spinules on ventral surface between veins $R_{2+3}$ and $R_{4+5}$. Legs black except for all tarsomeres brownish. Fore femur with 6-7 setae on ventral surface. Mid tibia with 4 strong setae on basal part of dorsal surface. Abdominal tergites and sternites nearly entirely brown.

Male terminalia: Hypandrium anteromedially elongated, about 1.56 times as long as width of epandrium, without paramedian setae posteroventrally (Figs 29, 30). Gonopods narrow distally, subacute apically (Fig. 28). Aedeagus lacks serrated, minute processes, about 0.29 times as long as aedeagal apodeme (Figs 29, 30).

Measurements. $\mathrm{BL}=3.15 \mathrm{~mm}$ in holotype (range in $4 \sigma^{\dagger}$ and 5 paratypes: $2.90-3.65 \mathrm{~mm}$ in $\delta, 3.60-4.15 \mathrm{~mm}$ in + ), $\mathrm{ThL}=$ $1.64 \mathrm{~mm}(1.64-1.92 \mathrm{~mm}$ in $\widehat{\delta}, 1.86-2.20 \mathrm{~mm}$ in ㅇ $), \mathrm{WL}=2.80$ $\mathrm{mm}\left(2.75-3.35 \mathrm{~mm}\right.$ in $\delta, 3.40-4.1 \mathrm{~mm}$ in $\left.{ }^{\top}\right), \mathrm{WW}=1.20 \mathrm{~mm}$ 


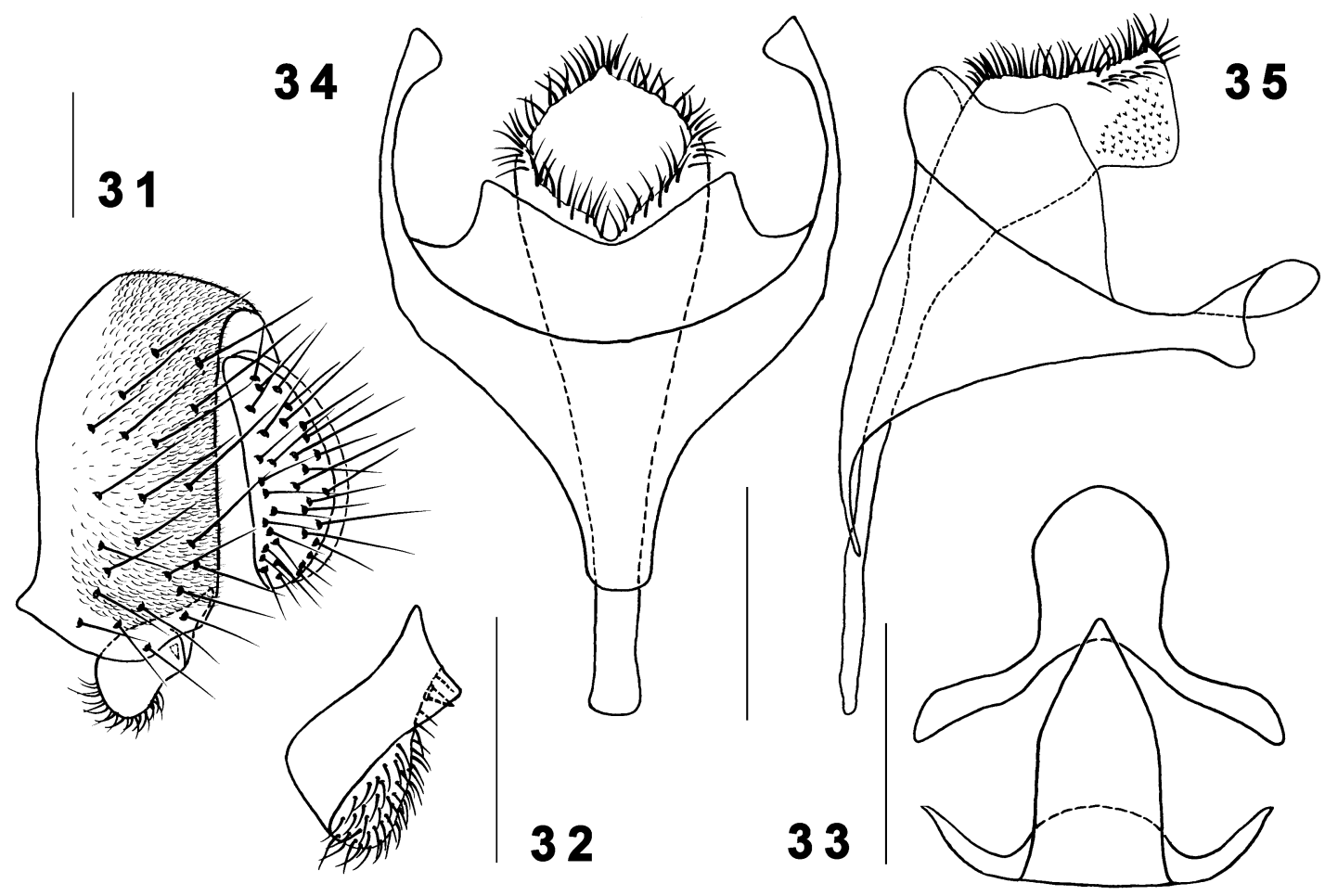

Figs 31-35. Stegana (Steganina) nigriclypeata Chen \& Chen, sp. n., ô. 31 - epandrium, surstylus and cercus; 32 - surstylus; 33 10th sternite and gonopods; 34 - hypandrium, aedeagus and aedeagal apodeme; 35 - ditto. For orientation, see Figs 3-7. Scale bars $=0.1 \mathrm{~mm}$.

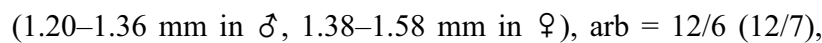
avd $=0.83(0.75-0.83)$, adf $=2.14(1.87-2.28)$, flw $=2.14$ $(1.87-2.28), \quad \mathrm{FW} / \mathrm{HW}=0.48 \quad(0.46-0.53), \quad \mathrm{ch} / \mathrm{o}=0.16$ $(0.15-0.17)$, prorb $=0.88(0.88-1.12)$, rcorb $=0.75(0.67-0.89)$, $\mathrm{vb}=0.69(0.70-0.76), \mathrm{dcl}=0.50(0.33-0.50)$, sctl $=1.44$ $(1.45-1.72)$, sterno $=0.80(0.78-0.86)$, orbito $=2.50$ $(2.20-2.50), \mathrm{dcp}=0.24(0.17-0.24), \mathrm{sctlp}=1.54(1.81-2.40), \mathrm{C}$ $=1.81(1.92-2.21), 4 \mathrm{c}=1.19(1.16-1.33), 4 \mathrm{v}=1.68$ $(1.71-2.11), 5 \mathrm{x}=2.00(1.33-1.91), \mathrm{ac}=18.5(13.00-23.00), \mathrm{M}$ $=0.65(0.54-0.64), \mathrm{C} 3 \mathrm{~F}=0.82(0.78-0.86)$.

Type material. Holotype $\widehat{\delta}$ (KPSP), MALAYSIA: Poring, Sabah, 16.iii.1999, tree trunk, M.J. Toda. Paratypes: MALAYSIA: $13 \delta, 7 q(50,2 q$ in ITBC; $40,2 q$ in KPSP; $20,2 q$ in SEHU; $20,1+$ in SCAU, Nos 120260-62), same data as holotype; 2 (KPSP), Mt. Kinabalu, Sabah, Malaysia, 10.-11.iii.1999, fallen logs, M.J. Toda.

Etymology. A combination of the Latin words flavum + scutellum, referring to the yellow scutellum.

Distribution. Malaysia (Sabah).

Stegana (Steganina) nigriclypeata Chen \& Chen, sp. n. (Figs 31-35)

Diagnosis. Differs from $S$. flavipalpata in having an aedeagus that protrudes posterodorsally, with serrated, minute processes covering the protrusion (Fig. 35).

Description. Male: Frons brown. Fronto-orbital plate orange yellow. Pedicel orange yellow; first flagellomere yellow on basal $2 / 3$, black on distal $1 / 3$. Face yellow, black on lower margin. Clypeus black. Palpus almost black. Mesonotum yellowish brown, without stripes and patches. Postpronotal lobe yellowish brown. Katepisternum dark brown. Scutellum yellow. Costal vein with 9-10 minute spinules on ventral surface between veins
$\mathrm{R}_{2+3}$ and $\mathrm{R}_{4+5}$. Legs greyish yellow, white on basal part of fore femur and distal part of mid tibia, black on distal part of fore femur and whole of mid and hind femora. Fore femur with 3 setae on distal part of ventral surface. Abdominal tergites nearly entirely brown, sternites yellow.

Male terminalia: Distal part of 10th sternite slightly rounded, 0.56 times as wide as long (Fig. 33). Hypandrium anteromedially slightly elongated, about 1.39 times as long as width of epandrium, without paramedian setae posteroventrally (Figs 34, 35). Gonopods almost triangular distally (Fig. 33). Aedeagus about 0.72 times as long as aedeagal apodeme (Figs 34, 35).

Measurements. $\mathrm{BL}=2.43 \mathrm{~mm}$ in holotype, $\mathrm{ThL}=1.12 \mathrm{~mm}$, $\mathrm{WL}=2.13 \mathrm{~mm}, \mathrm{WW}=1.20 \mathrm{~mm}, \mathrm{arb}=14 / 5$, avd $=0.82$, adf $=$ 0.84 , flw $=1.88, \mathrm{FW} / \mathrm{HW}=0.57, \mathrm{ch} / \mathrm{o}=0.20$, prorb $=1.29$, rcorb $=0.71, \mathrm{vb}=0.58, \mathrm{dcl}=0.37$, sctl $=2.13$, sterno $=1.33$, orbito $=2.14, \mathrm{dcp}=0.21, \mathrm{sctl} p=2.40, \mathrm{C}=2.18,4 \mathrm{c}=1.00,4 \mathrm{v}=$ $1.82,5 \mathrm{x}=1.81, \mathrm{ac}=22.00, \mathrm{M}=0.66, \mathrm{C} 3 \mathrm{~F}=0.87$.

Type material. Holotype $\widehat{0}$ (KPSP), MALAYSIA: Poring, Sabah, 29.xii.1998, fallen log, M.J. Toda.

Etymology. A combination of the Latin words niger + clypeus, referring to the black clypeus.

Distribution. Malaysia (Sabah).

\section{Stegana (Steganina) nigripalpata Chen \& Chen, sp. n.} (Figs 36-39)

Diagnosis. Differs from $S$. nigriclypeata in having the distal part of 10th sternite slightly oblong, 0.33 times as wide as long (Fig. 37), and the gonopods narrow distally, acute apically (Fig. 37). 


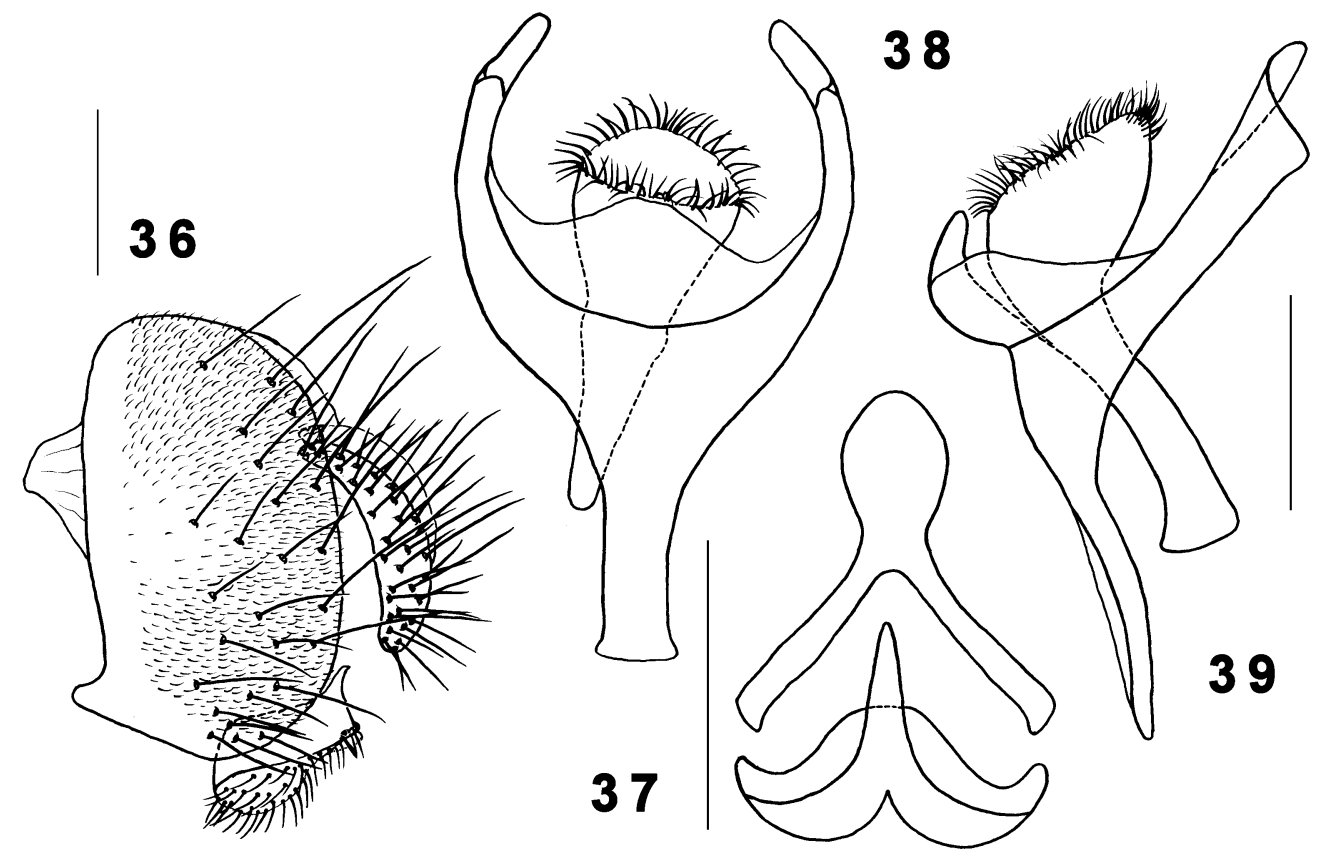

Figs 36-39. Stegana (Steganina) nigripalpata Chen \& Chen, sp. n., o. 36 - epandrium, surstylus and cercus; 37 - 10th sternite and gonopods; 38 - hypandrium, aedeagus and aedeagal apodeme; 39 - ditto. For orientation, see Figs 3-7. Scale bars $=0.1 \mathrm{~mm}$.

Description. Male. Frons brown. Fronto-orbital plate orange yellow. Pedicel orange yellow; first flagellomere yellow on basal 2/3, black on distal 1/3. Face yellow, brown on lower corners. Clypeus black. Palpus predominantly black. Mesonotum yellow, slightly brownish laterally, without stripes and patches. Postpronotal lobe yellowish brown. Katepisternum brown, slightly yellow ventrally. Scutellum yellow. Costal vein with 9 minute spinules on ventral surface between veins $R_{2+3}$ and $R_{4+5}$. Legs brown except for greyish yellow distal parts of mid and hind tibiae and all tarsomeres. Fore femur with 3 setae on distal part of ventral surface. Mid tibia with 6 strong setae on basal part of dorsal surface. Abdominal tergites and sternites nearly entirely brown.

Male terminalia: Hypandrium anteromedially elongated, about 1.67 times as long as width of epandrium, without paramedian setae (Figs 38, 39). Aedeagus without serrated, minute processes, about 0.7 times as long as aedeagal apodeme (Figs 38, 39).

Measurements. $\mathrm{BL}=2.74 \mathrm{~mm}$ in holotype, $\mathrm{ThL}=1.48 \mathrm{~mm}$, $\mathrm{WL}=2.56 \mathrm{~mm}, \mathrm{WW}=0.98 \mathrm{~mm}$, arb $=15 / 5$, avd $=0.92$, adf $=$ $1.50, \mathrm{flw}=1.88, \mathrm{FW} / \mathrm{HW}=0.47, \mathrm{ch} / \mathrm{o}=0.16$, prorb $=1.33$, rcorb $=0.67, \mathrm{vb}=0.50, \mathrm{dcl}=0.60, \mathrm{sctl}=1.70$, sterno $=1.33$, orbito $=2.00, \mathrm{dcp}=0.15, \mathrm{sctlp}=2.00, \mathrm{C}=1.97,4 \mathrm{c}=1.10,4 \mathrm{v}=$ $1.97,5 \mathrm{x}=1.90, \mathrm{ac}=32.00, \mathrm{M}=0.66, \mathrm{C} 3 \mathrm{~F}=0.74$.

Type material. Holotype $\hat{o}$ (KPSP), MALAYSIA: Poring, Sabah, 16.iii.1999, tree trunk, M.J. Toda.

Etymology. A combination of the Latin words niger + palpus, referring to the black palpus.

Distribution. Malaysia (Sabah).

Stegana (Steganina) melanothorax Chen \& Chen, sp. n. (Figs 40-44)

Diagnosis. Differs from S. leucothorax in having an entirely black clypeus and katepisternum and predominantly black palpus.
Description. Male and female. Frons black, with an orange yellow band submedially. Fronto-orbital plate orange yellow. Pedicel orange yellow; first flagellomere almost black. Face black, yellow on lower margin. Mesonotum dark brown, laterally with yellow stripes and patches. Postpronotal lobe brown. Scutellum brown. Costal vein with 7-8 minute spinules on ventral surface between veins $R_{2+3}$ and $R_{4+5}$. Legs dark brown, whitish yellow on distal parts of mid and hind tibiae, greyish yellow on all 2 nd to 5 th tarsomeres. Fore femur with 4-5 setae on distal part of ventral surface. Mid tibia with 4-5 strong setae on basal part of dorsal surface. Abdominal tergites and sternites nearly entirely dark brown.

Male terminalia. Distal part of 10th sternite slightly oblong, 0.8 times as wide as long (Fig. 42). Hypandrium anteromedially elongated, about 1.3 times as long as width of epandrium, without paramedian setae (Figs 43, 44). Gonopods roughly triangular distally, with narrowly rounded apex (Fig. 42). Aedeagus without serrated, minute processes, about 0.64 times as long as aedeagal apodeme (Figs 43, 44).

Measurements. $\mathrm{BL}=3.27 \mathrm{~mm}$ in holotype (range in $3 \sigma^{\dagger}$ and 3 paratypes: $2.88-3.36 \mathrm{~mm}$ in $\delta, 2.84-3.28 \mathrm{~mm}$ in + ), $\mathrm{ThL}=$ $1.68 \mathrm{~mm}(1.59-1.72 \mathrm{~mm}$ in $\delta, 1.42-1.78 \mathrm{~mm}$ in क $), \mathrm{WL}=3.40$ $\mathrm{mm}\left(2.76-3.36 \mathrm{~mm}\right.$ in $\delta^{\star}, 2.53-2.93 \mathrm{~mm}$ in + ), WW $=1.52 \mathrm{~mm}$ $(1.32-1.50 \mathrm{~mm}$ in $\delta, 1.24-1.47 \mathrm{~mm}$ in o $)$, arb $=12 / 8$ $(12-13 / 7-8)$, avd $=0.92(0.77-0.92)$, adf $=1.85(1.67-2.00)$, $\mathrm{flw}=1.85(1.71-2.33), \mathrm{FW} / \mathrm{HW}=0.43(0.40-0.46), \mathrm{ch} / \mathrm{o}=0.17$ $(0.15-0.17)$, prorb $=0.78(0.71-0.94)$, rcorb $=0.61(0.57-0.94)$, $\mathrm{vb}=0.69(0.65-0.76), \mathrm{dcl}=0.80(0.43-0.81)$, sctl $=1.80$ $(1.06-1.81)$, sterno $=0.74(0.74-0.94)$, orbito $=1.70$ $(1.67-2.20), \mathrm{dcp}=0.17(0.15-0.24), \mathrm{sctlp}=2.33(1.45-2.29), \mathrm{C}$ $=2.02(1.79-2.17), 4 \mathrm{c}=1.24(1.00-1.26), 4 \mathrm{v}=1.94$ $(1.25-1.85), 5 \mathrm{x}=1.27(1.21-1.50), \mathrm{ac}=20.50(18.50-20.50), \mathrm{M}$ $=0.58(0.47-0.61), \mathrm{C} 3 \mathrm{~F}=0.64(0.58-0.73)$. 




Figs 40-44. Stegana (Steganina) melanothorax Chen \& Chen, sp. n., o. 40 - epandrium, surstylus and cercus; 41 - surstylus; 42 10 th sternite and gonopods; 43 - hypandrium, aedeagus and aedeagal apodeme; 44 - ditto. For orientation, see Figs 3-7. Scale bars $=0.1 \mathrm{~mm}$.

Type material. Holotype o (SCAU, No. 120263), CHINA: Wangtianshu, Mengla, Xishuangbanna, Yunnan, 10.ix.2002, tree trunk, H.W. Chen. Paratypes: CHINA: $2 q$, same data as holotype (SCAU, Nos 120264, 65); 30, 19 (10, 19 in KIZ, 20 in SCAU, Nos 120266, 67), Menglun, Xishuangbanna, Yunnan, 13.-14.ix.2002, tree trunks, H.W. Chen.

Etymology. A combination of the Greek words melas + thorax, referring to the black katepisternum.

Distribution. China (Yunnan).

\section{Key to the species of the undulata group}

1 Surstylus mostly with 1 small acute prensiseta submedially on inner margin (absent in S. melanothorax), only distally with dense setae; distal part of 10th sternite expanded to lobate (the undulata group). . . . . . . . . . . 2

- Surstylus usually without or with only 1 prensiseta apically, or (in the shirozui group) with 1 row of 5-8 prensisetae submedially on inner margin, with several setae basally and sparse setae distally; distal part of 10th sternite not expanded. ................ other Steganina species

2 Veins $R_{4+5}$ and $M_{1}$ with several setae on basal section of dorsal surface. .............. undulata de Meijere

- Veins $\mathrm{R}_{4+5}$ and $\mathrm{M}_{1}$ without setae on basal section. ..... 3

3 Katepisternum white to white-yellow. ............ 4

- Katepisternum brown to black, sometimes pale ventrally. . 7

4 Face black, with yellow band medially; distal part of 10th sternite nearly rounded, its length distinctly greater than width. ........... flaviclypeata Chen \& Chen, sp. n.

- Face black on upper 1/2, yellow on lower 1/2; distal part of 10th sternite broadened, distinctly shorter than wide. . . . .5

5 Palpus entirely yellow; scutellum yellow with brown stripes or patches in male, dark brown with yellow tip in female; hypandrium with paramedian setae posteroventrally; aedeagus with numerous, serrated, minute processes. ...... ............... S. flavipalpata Chen \& Chen, sp. n. - Palpus yellow basally, black distally; scutellum brown; hypandrium without paramedian setae; aedeagus without numerous, serrated, minute processes. . . . . . . . . 6

6 Distal part of 10th sternite 1.9 times as wide as long; gonopods slightly triangular; distal part of aedeagus slightly

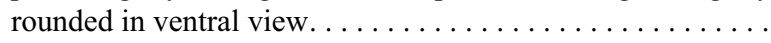
............ S. melanocheilota Chen \& Chen, sp. n.

- Distal part of 10th sternite 1.7 times as wide as long; gonopods shovel-like, paramedially parallel but acute apically; distal part of aedeagus triangular in ventral view. ........ S. leucothorax Chen \& Chen, sp. n.

7 First flagellomere almost black; face black, yellow on lower margin; scutellum brown. . . . . . . . . . . . . . . ............ S. melanothorax Chen \& Chen, sp. n. - First flagellomere yellow basally, black distally; face yellow, black on lower margin or corners; scutellum yellow.

............................... 8

8 Mesonotum yellow, with distinct dark brown stripes and patches laterally; fore femur with 6-7 setae on ventral surface; surstylus lacking prensiseta. . . . . . . . . . . . . ............. S. flaviscutellata Chen \& Chen, sp. n. - Mesonotum yellow, without distinct dark brown stripes and patches laterally; fore femur with 3 setae on distal part of ventral surface.................... 9

9 Katepisternum dark brown; aedeagus with numerous, serrated, minute processes apically. . . . . . . . . . . . $\ldots \ldots \ldots \ldots \ldots$ S. nigriclypeata Chen \& Chen, sp. n.

- Katepisternum brown, slightly yellowish ventrally; aedeagus without serrated, minute processes .......... $\ldots \ldots \ldots \ldots \ldots$ S. nigripalpata Chen \& Chen, sp. n. 


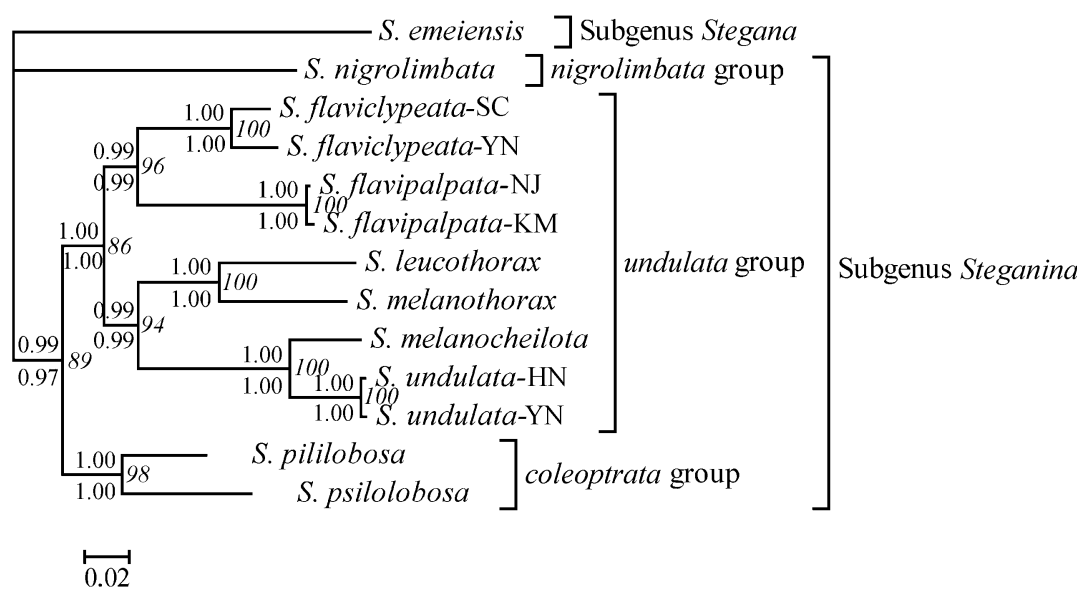

Fig. 45. Bayesian tree constructed using the concatenated sequences (2-partition scheme). Numbers above and below the branches indicate the posterior probabilities from 2- and 6-partition schemes, respectively; those to the right of the nodes (all shown in italics) are bootstrap percentages (1000 replicates) from the ME analysis.

\section{MOLECULAR PHYLOGENY}

\section{Data summary}

The alignment of the ND2 sequences spans 1029 sites, with 210 parsimony informative (PI) out of 350 variable sites; the alignment of the COI sequences spans 710 sites, with 134 PI out of 182 variable sites. There are end gaps in the ND2 sequences of $S$. melanothorax (sites 1-4) and S. undulata-YN (sites 1-11), and the COI sequences of $S$. melanocheilota (sites 1030-1043). Alignment gaps of three nucleotides were also found in the ND2 sequences of $S$. emeiensis and $S$. nigrolimbata (both at sites 268-270).

The result of the tests using STATIO is shown in Table 2 . The homogeneity was rejected for the partition of the 3 rd codon position of the ND2 sequences $(P=0.24 \%)$ and all the $N D 2+C O I$ sequences $(P=3.14 \%)$. As shown in the same table, the test using DAMBE yielded Iss indices significantly lower than the corresponding Iss.C index for the concatenated sequences or each of the partitions for a symmetrical tree, and the the Iss.c indices for an extreme asymmetric tree, except for the partition of the 3rd codon position of the ND2+COI sequences $(P=$ 0.3736). Since none of the resultant trees in the present study are extremely asymmetric, there should be little substitution saturation in the sequences used in this study.

\section{Phylogenetic reconstruction}

The 2- and 6-partition Bayesian and the ME analyses yielded the same tree topology, therefore only the 2-partition tree is shown, with the node supports of each of the analyses shown on this tree. The monophyly of the undulata group was well-supported [bootstrap percentage (BP) in the $\mathrm{ME}$ analysis $=86$; posterior probabilities (PPs) in the 2-/6-partiton Bayesian analyses $=1.00 / 1.00]$ as was the coleoptrata group $(\mathrm{BP}=98 ; \mathrm{PPs}=1.00 / 1.00)$. These two were sister groups $(\mathrm{BP}=89$; $\mathrm{PPs}=0.99 / 0.97)$, while the three groups of the subgenus Steganina did not form a monophyletic assemblage with respect to the subgenus Stegana. Within the undulata group, S. flaviclypeata and $S$. flavipalpata formed a monophyletic cluster $(\mathrm{BP}=96$; PPs $=0.99 / 0.99)$ and so $\operatorname{did} S$. leucothorax, S. melanothorax, S. melanocheilota and S. undulata $(\mathrm{BP}=94$; PPs $=0.99 / 0.99)$. Within the latter cluster, two species pairs were recovered: $S$. leucothorax $+S$. melanothorax $(\mathrm{BP}=100 ; \mathrm{PPs}=1.00 / 1.00)$ and $S$. undulata + S. melanocheilota $(\mathrm{BP}=100 ; \mathrm{PPs}=1.00 / 1.00)$.

\section{DISCUSSION}

\section{Base composition heterogeneity and phylogenetic relationship}

It is critical in phylogenetic reconstruction to account for among-taxa base composition heterogeneity, because sequences of similar base compositions are prone to be clustered erroneously (Tarrío et al., 2001). In the present study, the base composition homogeneity was rejected in the STATIO test at either the 3rd codon position of the $N D 2$ sequences $(P=0.24 \%)$ or the $N D 2+C O I$ data set as a whole $(P=3.14 \%)$. However, according to the results of this phylogenetic analyses, the base composition heterogeneity in the sequence data may be too limited to conceal the phylogenetic signals harboured in the sequences, since the phylogenetic analyses with either ME (taking into account the heterogeneous pattern among lineages) or Bayesian (not taking into account the heterogeneous pattern) methods recovered the same relationship among the taxa studied, and both methods yielded wellresolved relationships within the subgenus Steganina, except for the phylogenetic position of $S$. nigrolimbata.

\section{Species delimitation}

Meier et al. (2008) estimated the mean intraspecific variability, as well as the mean and smallest interspecific variabilities (\%) of the mitochondrial COI sequences in Diptera. The estimates were $1.3 \% \pm 1.6 \%, 10.1 \% \pm 3.0 \%$ and $5.9 \% \pm 4.1 \%$, respectively. In this study, the genetic distances between the ND2 sequences of both $S$. undulata (0.002) and $S$. flavipalpata (0.005) are very small; the COI sequences of the Nanjian (NJ) and Kunming (KM) samples of $S$. flavipalpata are identical. These distances are below the lower limit of the interval of the smallest interspecific COI variability of Meier et al. (2008). This 
corroborated the respective morphological similarities and strongly supports a conspecific status in both cases. As for S. flaviclypeata, a total of 30 or 25 nucleotide substitutions were found between the ND2 or COI sequences of its Sichuan (SC) and Yunnan (YN) samples. The equivalent p-distances $(0.029 / 0.035)$ are comparable to the upper limit of the mean intraspecific variability of mitochondrial COI sequence estimated by Meier et al. (2008), and falls in the estimate interval of the smallest interspecific variability. Actually, the p-distance of 0.029 in the ND2 sequences is smaller than those among some very closely related Drosophila species (0.036-0.045), but larger than some intraspecific ND2 sequence divergences in the genus Paraleucophenga (Zhao et al., 2009 and references therein). In this regard, the genetic distance between the two samples of S. flaviclypeata is likely to be of intraspecific level. However, the intra- and interspecific genetic distances may extensively overlap in Diptera (Meier et al., 2006), indicating that no universal cut-off criteria exist for determining the species status of dipteran species (DeSalle et al., 2005). Due to sparse taxon sampling at either the intra- or interspecific level in these molecular analyses, it is difficult to establish an objective threshold of distance for species diagnosis in the undulata group. In addition, currently there are only slight differences in the morphology recorded between the two local samples of $S$. flaviclypeata. Therefore, it may be premature to judge the significance of the differences between the samples of S. flaviclypeata.

ACKNOWLEDGEMENTS. We thank M.J. Toda for providing specimens, J. Máca for comments, M.B. Mohamed, M.B. Lakim, F. Liew, J. Nais, F. Zhao, H.L. Cao, H.Z. Cao and T. Li for help with fieldwork. This study was funded by a Grantin-Aid for Scientific Research from Japan Society for the Promotion of Science (No. 15255006) and the National Natural Science Foundation of China (Nos 30760037, 30970396).

\section{REFERENCES}

Bächli G., Vilela C.R., Andersson S. \& Saura A. 2004: The Drosophilidae (Diptera) of Fennoscandia and Denmark. Fauna Entomologica Scandinavica, Vol. 39. Brill, Leiden and Boston, $362 \mathrm{pp}$.

Brake I. \& BÄChli G. 2008: Drosophilidae (Diptera). World Catalogue of Insects, Vol. 9. Apollo Books, Stenstrup, 412 pp.

CAO H.Z. \& ChEN H.W. 2008: Revision of the Stegana nigrolimbata species group (Diptera: Drosophilidae) from the Oriental Region. Zootaxa 1848: 27-36.

Chen H.W. \& Toda M.J. 2001: A revision of the Asian and European species in the subgenus Amiota Loew (Diptera: Drosophilidae) and establishment of species-groups based on phylogenetic analysis. J. Nat. Hist. 35: 1517-1563.

Chen X.P. \& Chen H.W. 2008: The Stegana coleoptrata species group (Diptera: Drosophilidae) from mainland China. Zootaxa 1891: 55-65.

Chen X.P. \& Chen H.W. 2009a: Four new species of the subgenus Steganina (Diptera: Drosophilidae) from southern China. Ann. Zool. 59: 495-501.

Chen X.P. \& Chen H.W. 2009b: Five new species of the subgenus Stegana (Steganina) (Diptera: Drosophilidae) from East Malaysia. Orient. Insects 43: 229-239.
Chen X.P., Gao J.J. \& Chen H.W. 2009: The Stegana shirozui species group (Diptera: Drosophilidae). J. Nat. Hist. 43: 1909-1927.

Cheng Y., Gao J.J. \& Chen H.W. 2009: The Stegana ornatipes species group from the Oriental Region (Diptera: Drosophilidae). Zootaxa 2216: 37-48.

DE MeiJere J.C.H. 1911: Studien über südostasiatische Dipteren. VI. Tijdschr. Entomol. 54: 258-432.

DeSalle R., Egan C.G. \& Siddall M. 2005: The unholy trinity: taxonomy, species delimitation and DNA barcoding. Philos. Tr. Soc. (B) 360: 1905-1916.

DudA O. 1924: Beitrag zur Systematik der Drosophiliden unter besonderer Berücksichtigung der paläaktischen u. orientalischen Arten (Dipteren). Arch. Naturg. 90: 172-234.

Folmer O., Black M., Hoen W., Lutz R. \& Vrijenhoek R. 1994: DNA primers for amplification of mitochondrial cytochrome c oxidase subunit I from diverse metazoan invertebrates. Mol. Mar. Biol. Biot. 3: 294-299.

He X.F., Gao J.J., Cao H.Z., Zhang X.L. \& Chen H.W. 2009. Taxonomy and molecular phylogeny of the Phortica hani species complex (Diptera: Drosophilidae). Zool. J. Linn. Soc. 157: 359-372.

LAŠTovKa P. \& MÁcA J. 1982: European and North American species of the genus Stegana (Diptera, Drosophilidae). Annot. Zool. Bot. (Bratislava) 149: 1-38.

Li T., CaO H.Z., Gao J.J. \& Chen H.W. 2010: A revision of the subgenus Stegana (s. str.) (Diptera: Drosophilidae) from mainland China. Zool. J. Linn. Soc. 158: 726-739.

McAlpine J.F. 1981: Morphology and terminology: adults. In McAlpine J.F. (ed.): Manual of Nearctic Diptera 1. Research Branch Agriculture Canada Monograph 27. Research Branch Agriculture Canada, Ottawa, pp. 9-64.

Meier R., Shiyang K., Vaidya G. \& NG P.K.L. 2006: DNA barcoding and taxonomy in Diptera: a tale of high intraspecific variability and low identification success. Syst. Biol. 55: 715-728.

Meier R., Zhang G. \& Ali F. 2008: The use of mean instead of smallest intraspecific distances exaggerates the size of the "barcoding gap" and leads to misidentification. Syst. Biol. 57: 809-813.

Meigen J.W. 1830: Systematische Beschreibung der bekannten europäischen zweiflügeligen Insekten. Vol. 6. Schulze, Hamm, 401 pp.

OKADA T. 1971: A revision and taxometric analysis of the genus Stegana Meigen of Japan and adjacent countries (Diptera: Drosophilidae). Mushi 45: 81-99.

OKADA T. \& Sidorenko V.S. 1992: New or unrecorded species of Stegana (Steganina) (Diptera: Drosophilidae) from the Old World, especially Japan and Russia. Jap. J. Entomol. 60: 415-426.

Otranto D., Stevens J.R., Testini G., Cantacessi C. \& Máca J. 2008: Molecular characterization and phylogenesis of Steganinae (Diptera: Drosophilidae) inferred by the mitochondrial cytochrome c oxidase subunit 1. Med. Vet. Entomol. 22: 37-47.

PARK J. 1999: Molecular Phylogenetic Studies of the Drosophila (Drosophila) virilis Section (Diptera: Drosophilidae). Ph.D. Thesis, Tokyo Metropolitan University, 54 pp.

Posada D. \& Crandall K.P. 1998: Modeltest: testing the model of DNA substitution. Bioinformatics 14: 817-818.

RzHETSKy A. \& NeI M. 1995: Tests of applicability of several substitution models for DNA sequence data. Mol. Biol. Evol. 12: 131-151. 
Ronquist F. \& Huelsenbeck J.P. 2003: MrBayes 3: Bayesian phylogenetic inference under mixed models. Bioinformatics 19: $1572-1574$.

Sidorenko V.S. 1997: New Asian species and new records of the genus Stegana Meigen (Diptera: Drosophilidae). I. Subgenera Oxyphortica Duda and Stegana s. str. Ann. Soc. Entomol. Fr. 33: 65-79.

Sidorenko V.S. 1998: New Asian species and new records of the genus Stegana Meigen (Diptera: Drosophilidae). III. Descriptions, taxonomic remarks and key to the Asian species. Ann. Soc. Entomol. Fr. 34: 285-300.

Sidorenko V.S. 2002: Phylogeny of the tribe Steganini Hendel and some related taxa (Diptera: Drosophilidae). Far East. Entomol. 111: 1-20.

Sidorenko V.S. \& OKadA T. 1991: Descriptions of three new species of Stegana (Stegana) Meigen (Diptera: Drosophilidae) from Sichuan Province, China, and Formosa, with taxometric analyses of the subgenus. Jap. J. Entomol. 59: 655-662.

Tamura K., Dudley J., Nei M. \& Kumar S. 2007: MEGA4: Molecular Evolutionary Genetics Analysis (MEGA) software version 4.0. Mol. Biol. Evol. 24: 1596-1599.

Tarrío R., Rodríguez-Trelles F. \& Ayala F.J. 2001: Shared nucleotide composition biases among species and their impact on phylogenetic reconstructions of the Drosophilidae. Mol. Biol. Evol. 18: 1464-1473.

Thompson J.D., Higgins D.G. \& Gibson T.J. 1994: Clustal W: improving the sensitivity of progressive multiple sequence alignment through sequence weighting, position specific gap penalties and weight matrix choice. Nucleic Acids Res. 22: 4673-4680.

Wang B.C., Park J., Watabe H., Gao J.J., Xiangyu J.G., AotsuKa T., Chen H.W. \& Zhang Y.P. 2006. Molecular phylogeny of the Drosophila virilis section (Diptera: Drosophilidae) based on mitochondrial and nuclear sequences. Mol. Phylogenet. Evol. 40: 484-500.

WheELER M.R. 1960: A new subgenus and species of Stegana Meigen (Diptera: Drosophilidae). Proc. Entomol. Soc. Wash. 62: 109-111.

XIA X. \& XIE Z. 2001: DAMBE: data analysis in molecular biology and evolution. J. Hered. 92: 371-373.

Xia X.H., XIe Z.H., Salemi M., Chen L. \& Wang Y. 2003: An index of substitution saturation and its application. Mol. Phylogenet. Evol. 26: 1-7.

Zhang W.X. \& ToDA M.J. 1992: A new species-subgroup of the Drosophila immigrans species-group (Diptera: Drosophilidae), with description of two new species from China and revision of taxonomic terminology. Jap. J. Entomol. 60: 839-850.

Zhao F., Gao J.J. \& Chen H.W. 2009: Taxonomy and molecular phylogeny of the Asian Paraleucophenga Hendel (Diptera: Drosophilidae). Zool. J. Linn. Soc. 155: 616-629.

Received July 9, 2010; revised and accepted August 16, 2010 